\title{
TURING BIFURCATION IN NONLINEAR COMPETITION MODELS WITH DELAY
}

\author{
BY
}

\author{
S. ROY CHOUDHURY AND C. FOSSER
}

Department of Mathematics, University of Central Florida, Orlando

\begin{abstract}
Turing instability in reaction-diffusion and predator-prey models including diffusion and Volterra-type distributed delays in the interspecies interaction terms is considered. For general functional forms of the reaction terms/prey birth ratepredator death rate, and delays modeled by the "weak" generic kernel $a \exp (-a U)$ and the "strong" generic kernel $a^{2} U \exp (-a U)$, the necessary and sufficient conditions for Turing instability are derived and are found to be significantly different from the classical conditions with no delay. The structure of the resulting Turing space, where steady states may be diffusionally driven unstable initiating spatial patterns, is delineated for four specific models, and compared to the corresponding regimes in the absence of delay. An alternative bifurcation-theoretic derivation of the boundary of the Turing-unstable domain is also presented.
\end{abstract}

1. Introduction. The onset of diffusive instability as a cause for the occurrence of spatial form and pattern from a spatially homogeneous initial state has been extensively pursued in the study of morphogenesis. The origins of this subject are due to Turing [1], who in the 1950s showed how diffusion may, somewhat counterintuitively, lead not to homogeneous final states but rather to areas of concentration of each of the reactants. Turing studied reaction-diffusion equations (in the setting of chemical kinetics) of the general form:

$$
\begin{aligned}
\frac{\partial C}{\partial t} & =R_{1}\left(C_{1}, C_{2}\right)+D_{1} \frac{\partial^{2} C_{1}}{\partial x^{2}}, \\
\frac{\partial C_{2}}{\partial t} & =R_{2}\left(C_{1}, C_{2}\right)+D_{2} \frac{\partial^{2} C_{2}}{\partial x^{2}}
\end{aligned}
$$

where

$$
C_{1}=C_{1}(x, t), \quad C_{2}=C_{2}(x, t)
$$

represent the amount or concentration of each reactant.

Received May 17, 1993.

1991 Mathematics Subject Classification. Primary 35B35, 45K05, 80A30, 92C15.

Key words and phrases. Turing bifurcations or instability, delay, reaction-diffusion and predator-prey models.

(C)1996 Brown University 
Let

$$
\begin{aligned}
R_{1}\left(C_{1}, C_{2}\right) & =\text { rate of production (or growth) of } C_{1}, \\
R_{2}\left(C_{1}, C_{2}\right) & =\text { rate of production (or growth) of } C_{2}, \\
D_{1} & =\text { diffusion coefficient of } C_{1}, \\
D_{2} & =\text { diffusion coefficient of } C_{2} .
\end{aligned}
$$

Turing found that, independent of the form of the reaction functions $R_{1}$ and $R_{2}$, diffusion could destabilize an otherwise stable equilibrium. This results in nonuniform spatial patterns, referred to as pre-pattern. This mechanism for the formation of pre-pattern in the embryonic stage of development could later lead to biological patterns by gene activation. Various extensions, applications, and quantitative verifications of the theory have since occurred. These include early theoretical and analytical extensions [2-4] and applications in developmental biology stimulated by the work of Meinhardt and Gierer [5-7] who performed numerical simulations of reaction-diffusion systems in various geometries. Segel and Jackson [4] extended the concept to macroscopic (Lotka-Volterra) models in population biology by showing how spatial patterns may occur, in particular for species dispersing at different rates. Investigations of nonstationary (traveling-wave) patterns, spirals, solitary peaks, fronts, and bifurcation analysis [8-12] have analytically confirmed and extended the work of Meinhardt and Gierer [5-7], and are reviewed in [13]. In [1417] chemical reactions and geometric considerations are considered as explanations for the properties of spatial patterns. Other explanations of pattern formation, not based on reaction-diffusion equations and the Turing mechanism, have also been investigated [18]. Reviews of all of these and other related works have been compiled separately by Levin and Segel [19], Murray [20], and Edelstein-Keshet [21].

In this paper we shall consider Turing instabilities in generalized chemical and biological models. Since at least one of the physical variables may depend on the past history of the system, we will incorporate such effects by introducing time delays into the equations. Factors that introduce time lags may include resource regeneration time, food storage or ingestion delays, maturation periods (thresholds), feeding times and hunger coefficients in predator-prey interactions, reaction times, and age structure of the population (influencing the birth and death rates). MacDonald [22] extensively reviews models incorporating time delays in diverse spatially-homogeneous biological models, and Cushing [23] does the same in the context of predator-prey models. Among the time-delay models considered in these reviews are the continuous models suggested by Kolmogorov, May, Holing, Hsu, Leslie, and Caperon, and others, as well as various discrete models.

The general form of the Volterra or convolution time delay is given by

$$
Q(x, t)=\int_{-\infty}^{t} C(x ; \tau) \widehat{G}(t-\tau) d \tau
$$

where $\widehat{G}(U)$ is the memory function or delay kernel [22, 23]. Notice that for the memory function $\widehat{G}(U)=\delta(U)$, one gets the case of the so-called discrete (or zero) delay. 
The effects of the delay may be treated [23-25] in the setting of functional differential equations for spatially homogeneous models. Since it has been shown [22, 26] that the "linear chain" form of the time delay has been very useful (the origin of this in the theory of elasticity is discussed in [22] and in the work of Vogel [25]) we will employ this type of delay in this investigation. The "linear chain" form of the memory function is

$$
\widehat{G}(U)=G_{a}^{p}(U)=\frac{a^{p+1} U^{p}}{p !} e^{-a U},
$$

where $1 / a$ measures the influence of the past, which decreases as $a$ increases.

The two most common types of delay kernels are when $p=0$, and when $p=1$. The "weak" $\left(G_{a}^{0}(U)\right)$ and "strong" $\left(G_{a}^{1}(U)\right)$ generic kernels [23] have been used in the context of various spatially homogeneous predator-prey models with delay [23, $24,27]$, Physically, the "weak" generic kernel $G_{a}^{0}(U)$ and "strong" generic kernel $G_{a}^{1}(U)$ have quite different behavior in terms of modeling the memory (or delay) effects. While the former decays monotonically for $U<0$, and represents a memory that progressively becomes weaker as one goes further into the past, the latter increases for a range $U<1 / a$. Although they may possibly be quantitatively different, it turns out that the effects of the "strong" delay on the regimes of instability are qualitatively similar to using the "weak" delay. Therefore, we will primarily model delay effects on Turing bifurcations in general reaction-diffusion systems using the "weak" generic kernel and make occasional references to the results using the "strong" kernel. An integrodifferential system is created by introducing the time delay(s) into the differential system (1). The "weak" generic kernel, $G_{a}^{0}$, reduces the integrodifferential system to a differential system with $Q$ replacing the variable $C$ and the added linear equation [22]:

$$
\frac{\partial Q}{\partial t}=a(C-Q)=a C-a Q .
$$

We will analyze delay in four models. The first is a chemical kinetics model for biological morphogenesis proposed by Gierer and Meinhardt [7]. Consider (1) with

$$
\begin{aligned}
& R_{a}(a, h)=\frac{c_{1} a^{2}}{h}-a \mu, \\
& R_{h}(a, h)=c_{2} a^{2}-\nu h
\end{aligned}
$$

where $C_{1}=a, C_{2}=h$, and $a(t)$ and $h(t)$ are the concentrations of the activator and inhibitor, respectively. Now consider the modification of system (1) with (5) by introducing a distributed (Volterra or convolution-type [23]) delay in the interspecies interaction term in the first equation (5a). The resultant system is (1) together with

$$
\begin{aligned}
& R_{a}(a, h)=\frac{c_{1} a}{h} \int_{-\infty}^{t} a(\tau) \widehat{G}(t-\tau) d \tau-a \mu, \\
& R_{h}(a, h)=c_{2} a^{2}-\nu h
\end{aligned}
$$

and will subsequently be referred to as GM-I. Here the time delay is introduced into Eq. (6a) in such a way that the activator may affect its own activation rate. There 
may be systems where the activator's effect on its own activation rate also affects the inhibitor's concentration; so we will consider delay in both equations. This results in the system (1) together with

$$
\begin{aligned}
& R_{a}(a, h)=\frac{c_{1} a}{h} \int_{-\infty}^{t} a(\tau) \widehat{G}(t-\tau) d \tau-a \mu \\
& R_{h}(a, h)=c_{2} a \int_{-\infty}^{t} a(\tau) \widehat{G}(t-\tau) d \tau-\nu h
\end{aligned}
$$

and will be referred to as GM-II.

The second model that will be analyzed is the general predator-prey model described by Segel and Jackson in [4] which, again, is a specific model of the type in (1) with

$$
\begin{aligned}
& R_{V}(V, P)=V k_{0}+k_{1} V^{2}-A V P, \\
& R_{P}(V, P)=B V P-C P^{2}
\end{aligned}
$$

where $C_{1}=V, C_{2}=P$, and $V(t)$ and $P(t)$ denote the population densities of the victim (prey) and predator, respectively. Modifying this system by again placing the time delay in the interspecies interaction term in only the second equation, the resulting system is (1) with

$$
\begin{aligned}
& R_{V}(V, P)=V k_{0}+k_{1} V^{2}-A V P, \\
& R_{P}(V, P)=B P \int_{-\infty}^{t} V(\tau) \widehat{G}(t-\tau) d \tau-C P^{2}
\end{aligned}
$$

and will be referred to as SJ-I. By placing the time delay only in the equation representing the density of the predatory we are attempting to capture the effects of such things as reproduction and maturation time. Assuming that there are systems where, in addition to the interspecies interaction time lag affecting the density of the predator, the density of the prey (victim) after a time lag is also affected by its own density, the system becomes (1) with

$$
\begin{aligned}
& R_{V}(V, P)=V k_{0}+k_{1} V^{2}-A P \int_{-\infty}^{t} V(\tau) \widehat{G}(t-\tau) d \tau, \\
& R_{P}(V, P)=B P \int_{-\infty}^{t} V(\tau) \widehat{G}(t-\tau) d \tau-C P^{2}
\end{aligned}
$$

and will be referred to as SJ-II.

The third model that will be considered is the autocatalytic Schnackenberg system [14], which is a chemical kinetics model. This model will be analyzed in dimensionless form, which results in a slight variation of the general form (1) with the dimensionless kinetics

$$
\begin{aligned}
& R_{u}(u, \nu)=a-u+u^{2} \nu, \\
& R_{\nu}(u, \nu)=b-u^{2} \nu .
\end{aligned}
$$


Here the concentrations of $C_{1}$ and $C_{2}$ are associated with $u$ and $\nu$, respectively, so that the system without delay is

$$
\begin{aligned}
& \frac{\partial u}{\partial t}=R_{u}(u, \nu)+\frac{\partial^{2} u}{\partial x^{2}}, \\
& \frac{\partial \nu}{\partial t}=R_{\nu}(u, \nu)+D \frac{\partial^{2} \nu}{\partial x^{2}}
\end{aligned}
$$

where $D=D_{2} / D_{1}$. We will modify this system by placing time delays in the autocatalytic term in both equations resulting in system (1) with

$$
\begin{aligned}
& R_{u}(u, \nu)=a-u+u \nu \int_{-\infty}^{t} u(\tau) \widehat{G}(t-\tau) d \tau, \\
& R_{\nu}(u, \nu)=b-u \nu \int_{-\infty}^{t} u(\tau) \widehat{G}(t-\tau) d \tau .
\end{aligned}
$$

The final model that we will examine is the Thomas immobilized-enzyme substrateinhibition mechanism [14]. It involves the experimental arrangement of two chemicals, $S$ and $A$, diffusing through an inactive membrane of thickness $L_{1}$ with diffusion coefficients $D_{S}^{\prime}, D_{A}^{\prime}$ onto a membrane of thickness $L_{2}$ containing the immobilized enzyme. The chemicals diffuse on this membrane with diffusion coefficients $D_{S}$ and $D_{A}$ and react at the empirical rate

$$
J=V_{m} A S /\left(K_{m}+S+S^{2} / K_{S}\right),
$$

where $V_{m}, K_{m}$, and $K_{S}$ are constants, and $K_{S}$ is related to the substrate inhibition. The dimensionless form of the system is (1) with

$$
\begin{aligned}
& R_{u}(u, \nu)=a-u-\frac{\rho u \nu}{\left(1+u+K u^{2}\right)}, \\
& R_{\nu}(u, \nu)=\alpha(b-\nu)-\frac{\rho u \nu}{\left(1+u+K u^{2}\right)}
\end{aligned}
$$

where $C_{1}=S$ and $C_{2}=A$, and is associated with the dimensionless variables $u$ and $\nu$, respectively. We introduce time delay in the substrate-inhibition reaction rate term by placing it in the chemical interaction factor resulting in (1) with

$$
\begin{aligned}
& R_{u}(u, \nu)=a-u-\frac{\rho \nu \int_{-\infty}^{t} u(\tau) \widehat{G}(t-\tau) d \tau}{\left(1+u+K u^{2}\right)}, \\
& R_{\nu}(u, \nu)=\alpha(b-\nu)-\frac{\rho \nu \int_{-\infty}^{t} u(\tau) \widehat{G}(t-\tau) d \tau}{\left(1+u+K u^{2}\right)} .
\end{aligned}
$$

This will be referred to as the Thomas model.

The remainder of this paper is organized as follows. In Sec. 2 the Turing bifurcation (instability) analysis for the general reaction-diffusion system (1) with delays modeled by the "weak" kernel is carried out. The results of the classical analysis without delay are briefly summarized in Appendix A for comparison purposes, and the analysis using the "strong" generic kernel is contained in Appendix B. In Sec. 3, the quadratic and cubic polynomial conditions of Sec. 2 for the Turing instability are 
used to derive the necessary and sufficient conditions for instability in the presence of delay. These conditions are shown to be entirely different from those in the absence of delay, resulting in the regimes of Turing instability being, in general, dramatically altered by delay effects. The structure of unstable Turing space for each model with delay is explored in Sec. 4 for the GM-I, GM-II, SJ-I, SJ-II, Schnackenberg, and Thomas models. The boundary of the Turing unstable regime is also re-derived there via an alternative, and more direct, bifurcation-type analysis. A brief summary of the results and conclusions is given in Sec. 5 .

2. Turing instability analysis with delay. The linearized version of the general system (1) is

$$
\begin{aligned}
& \frac{\partial C_{1}^{\prime}}{\partial t}=a_{11} C_{1}^{\prime}+a_{12} C_{2}^{\prime}+D_{1} \frac{\partial^{2} C_{1}^{\prime}}{\partial x^{2}}, \\
& \frac{\partial C_{2}^{\prime}}{\partial t}=a_{21} C_{2}^{\prime}+a_{22} C_{2}^{\prime}+D_{2} \frac{\partial^{2} C_{2}^{\prime}}{\partial x^{2}}
\end{aligned}
$$

where

$$
a_{i j}=\frac{\partial R_{i}}{\partial C_{j}} \mid(i, j=1,2),
$$

and

$$
\begin{aligned}
& C_{1}^{\prime}=C_{1}(x, t)-\widehat{C}_{1}, \\
& C_{2}^{\prime}=C_{2}(x, t)-\widehat{C}_{2}
\end{aligned}
$$

are small nonhomogeneous perturbations of the positive spatially uniform steady state $\left(\widehat{C}_{1}, \widehat{C}_{2}\right)$.

If the effects of a delay are introduced on the quantity $C_{1}$ as in (4), the linearized system of differential equations becomes

$$
\begin{aligned}
& \frac{\partial C_{1}^{\prime}}{\partial t}=a_{11} C_{1}^{\prime}+a_{12} C_{2}^{\prime}+a_{13} Q+D_{1} \frac{\partial^{2} C_{1}^{\prime}}{\partial x^{2}}, \\
& \frac{\partial C_{2}^{\prime}}{\partial t}=a_{21} C_{2}^{\prime}+a_{22} C_{2}^{\prime}+a_{23} Q^{\prime}+D_{2} \frac{\partial^{2} C_{2}^{\prime}}{\partial x^{2}} \\
& \frac{\partial Q^{\prime}}{\partial t}=a_{31} C_{1}^{\prime}+a_{32} C_{2}^{\prime}+a_{33} Q^{\prime}
\end{aligned}
$$

where $a_{31}=a, a_{32}=0$, and $a_{33}=-a$. The partial differential system (7) is linear and may be solved by separation of variables and Fourier analysis [28] in the standard way. Since the various Fourier modes do not interact under the linear operators of (7), it will suffice to consider a single mode

$$
S^{\prime}=\left[\begin{array}{l}
C_{1}^{\prime} \\
C_{2}^{\prime} \\
Q^{\prime}
\end{array}\right]=\left[\begin{array}{l}
\alpha_{1} \\
\alpha_{2} \\
\alpha_{3}
\end{array}\right] e^{\sigma t} \cos (q x)
$$


Using this in (7), we have

$$
\begin{aligned}
\alpha_{1}\left(\sigma-a_{11}+D_{1} q^{2}\right)+\alpha_{2}\left(-a_{12}\right)+\alpha_{3}\left(-a_{13}\right) & =0, \\
\alpha_{1}\left(-a_{21}\right)+\alpha_{2}\left(\sigma-a_{22}+D_{2} q^{2}\right)+\alpha_{3}\left(-a_{23}\right) & =0, \\
\alpha_{1}(-a)+\alpha_{2}(0)+\alpha_{3}(\sigma+a) & =0 .
\end{aligned}
$$

A nontrivial solution only exists if the determinant of the coefficients appearing in (8) is zero, yielding

$$
\sigma^{3}+\beta_{1} \sigma^{2}+\beta_{2} \sigma+\beta_{3}=0
$$

where

$$
\begin{aligned}
\beta_{1}= & -a_{11}-a_{22}+a+D_{1} q^{2}+D_{2} q^{2}, \\
\beta_{2}= & -a a_{11}-a a_{13}-a_{21} a_{12}-a a_{22}+a_{11} a_{22}+a D_{1} q^{2} \\
& -a_{22} D_{1} q^{2}+a D_{2} q^{2}-a_{11} D_{2} q^{2}+D_{1} D_{2} q^{4}, \\
\beta_{3}= & -a a_{21} a_{12}+a a_{11} a_{22}+a a_{13} a_{22}-a a_{23} a_{12}-a a_{22} D_{1} q^{2} \\
& -a a_{11} D_{2} q^{2}-a a_{13} D_{2} q^{2}+a D_{1} D_{2} q^{4} .
\end{aligned}
$$

We wish to determine if for some wavenumber $q^{2}$, the eigenvalue $\sigma$ can have a positive real part. This would mean that perturbations of a particular "waviness" or wavelength could cause instability to occur.

3. Conditions for diffusive instability. In order to isolate diffusion as the destabilizing mechanism we will first determine under what conditions the system is stable in the absence of diffusion. This can be accomplished by setting $D_{1}=D_{2}=0$ in Eqs. (9) or (10). Using the Routh-Hurwitz criteria [20], the necessary and sufficient conditions for the characteristic equation to have $\operatorname{Re}\left(\sigma_{1,2,3}\right)<0$ in the absence of diffusion are

conditions for stability without diffusion

$$
\begin{array}{ll}
\text { 1. } & \beta_{1}>0, \\
\text { 2. } & \beta_{3}>0, \\
\text { 3. } & \beta_{1} \beta_{2}-\beta_{3}>0 .
\end{array}
$$

Then introducing diffusion $\left(D_{1} \neq D_{2} \neq 0\right)$ we analyze how the stability conditions may be violated. Violating any of the above stability conditions may cause the steadystate $\left(\widehat{C}_{1}, \widehat{C}_{2}\right)$ to become unstable. In other words, a regime of instability could be created for some $q^{2}$. Analyzing (23), we next determine the necessary and sufficient conditions for diffusive instability with "weak" delays. In the next section we will use these conditions to graph the parameter regimes leading to Turing-type instabilities. Additionally, the results will be compared to the case of no delay contained in Appendix A. 
Condition 1. $\left(\beta_{1}>0\right)$.

$$
H_{1}\left(q^{2}\right) \equiv-a_{11}-a_{22}+a+D_{1} q^{2}+D_{2} q^{2}>0 .
$$

Since $D_{1}, D_{2}$, and $q^{2}$ are all positive quantities, condition (24) cannot be violated as long as (23a) holds. The condition (23a) for stability without diffusion may be written

$$
H_{1}(0)>0
$$

Condition 2. $\left(\beta_{3}>0\right)$.

$$
\begin{aligned}
H_{2}\left(q^{2}\right)= & -a a_{21} a_{12}+a a_{11} a_{22}+a a_{13} a_{22}-a a_{23} a_{12}-a a_{22} D_{1} q^{2} \\
& -a a_{11} D_{2} q^{2}-a a_{13} D_{2} q^{2}+a D_{1} D_{2}\left(q^{2}\right)^{2}>0 .
\end{aligned}
$$

In order to have instability for certain values of $q^{2}$ we require that

$$
\mathrm{H}_{2}\left(q^{2}\right)<0 \text {. }
$$

$\mathrm{H}_{2}$ is a quadratic expression in $q^{2}$, with leading coefficient positive, implying a parabola with positive concavity, which has a minimum obtained by simple calculus

$$
q_{2, \min }^{2}=\frac{D_{1} a_{22}+D_{2}\left(a_{11}+a_{13}\right)}{2 D_{1} D_{2}} .
$$

For a physically meaningful wavenumber we require that $a_{2, \min }^{2}$ be positive, providing another condition for diffusive instability. Therefore, if $q_{2, \min }^{2}<0$, no instability, but if $q_{2, \min }^{2}>0$, then in order to satisfy (27) a minimal condition is that

$$
\begin{aligned}
H_{2}\left(q_{2, \min }^{2}\right)= & -a_{12} a_{21}+a_{11} a_{22}+a_{13} a_{22}+\frac{a_{11}\left(-D_{2} a_{11}-D_{2} a_{13}-D_{1} a_{22}\right)}{2 D_{1}} \\
& +\frac{a_{13}\left(-D_{2} a_{11}-D_{2} a_{13}-D_{1} a_{22}\right)}{2 D_{1}}+\frac{a_{22}\left(-D_{2} a_{11}-D_{2} a_{13}-D_{1} a_{22}\right)}{2 D_{2}} \\
& +\frac{\left(-D_{2} a_{11}-D_{2} a_{13}-D_{1} a_{22}\right)^{2}}{4 D_{1} D_{2}}-a_{12} a_{23}<0 .
\end{aligned}
$$

Notice that in (26) at every occurrence of $q^{2}$ either $D_{1}$ or $D_{2}$ always appears in the term, and that $D_{1}$ and $D_{2}$ never appear in a term not containing $q^{2}$. This means that $H_{2}(0)$ represents condition (23a) without diffusion and may be written as $H_{2}(0)>0$.

The following is a summary of the conditions resulting from the analysis of the quadratic $\mathrm{H}_{2}\left(q^{2}\right)$ :

(a) $H_{2}(0)<0-$ unstable without diffusion; so instability is not diffusion-driven;

(b) $H_{2}(0)>0, q_{2, \min }^{2}<0$-no instability;

(c) $\mathrm{H}_{2}(0)>0, q_{2, \min }^{2}>0, \mathrm{H}_{2}\left(q_{2, \min }^{2}\right)>0$-no instability;

(d) $H_{2}(0)>0, q_{2, \min }^{2}>0, H_{2}\left(q_{2, \min }^{2}\right)<0$-instability. 
Therefore, the first set of necessary and sufficient conditions for the occurrence of Turing instability using the "weak" generic kernel is

$$
\begin{aligned}
\text { I. } & H_{2}(0)>0, \\
\text { II. } & q_{2, \min }^{2}>0, \\
\text { III. } & H_{2}\left(q_{2, \min }^{2}\right)<0 .
\end{aligned}
$$

Condition 3. $\left(\beta_{1} \beta_{2}-\beta_{3}>0\right)$. This may be written as the cubic (with $z=q^{2}$ )

$$
H_{3}(z)=\alpha z^{3}+\beta z^{2}+\gamma z+\delta
$$

where

$$
\begin{aligned}
\alpha= & D_{1}^{2} D_{2}+D_{1} D_{2}^{2}, \\
\beta= & a D_{1}^{2}+2 a D_{1} D_{2}+a D_{2}^{2}-2 a D_{1} D_{2} a_{11}-D_{2}^{2} a_{11} \\
& -D_{1}^{2} a_{22}-2 D_{1} D_{2} a_{22}, \\
\gamma= & a^{2} D_{1}+a^{2} D_{2}-2 a D_{1} a_{11}-2 a D_{2} a_{11}+D_{2} a_{11}^{2} \\
& -a D_{1} a_{13}-D_{1} a_{12} a_{21}-D_{2} a_{12} a_{21}-2 a D_{1} a_{22} \\
& -2 a D_{2} a_{22}+2 D_{1} a_{11} a_{22}+2 D_{2} a_{11} a_{22}+D_{1} a_{22}^{2}, \\
\delta= & -\left(a^{2} a_{11}\right)+a a_{11}^{2}-a^{2} a_{13}+a a_{11} a_{13}+a_{11} a_{12} a_{21} \\
& -a^{2} a_{22}+2 a a_{11} a_{22}-a_{11}^{2} a_{22}+a_{22} a_{12} a_{21}+a a_{22}^{2} \\
& -a_{11} a_{22}^{2}+a a_{12} a_{23} .
\end{aligned}
$$

Notice that the coefficient of $\left(q^{2}\right)^{3}$ is $\left(D_{1} D_{2}^{2}+D_{1}^{2} D_{2}\right)$, which is always positive. We can say the following about $H_{3}\left(q^{2}\right)$ :

1. For $q^{2} \in \mathfrak{R}, q^{2} \rightarrow \infty$,

$$
H_{3}\left(q^{2}\right) \rightarrow \infty
$$

2. The first derivative is

$$
\frac{d H_{3}}{d z}=3 \alpha z^{2}+2 \beta z+\gamma,
$$

which is quadratic; so $H_{3}(z)$ has two local extrema at

$$
z_{1}=\frac{-\beta+\sqrt{\beta^{2}-3 \alpha \gamma}}{3 \alpha} \text { and } z_{2}=\frac{-\beta-\sqrt{\beta^{2}-3 \alpha \gamma}}{3 \alpha} .
$$


3. The second derivative is

$$
\frac{d^{2} H_{3}}{d z^{2}}=6 \alpha z+2 \beta
$$

whose dominant term has a positive coefficient, implying that for large $z \quad\left(=q^{2}\right)$, $\mathrm{H}_{3}$ is concave upwards.

4. If $z_{1}$ and $z_{2}$ are local extrema then (20) and (24) imply that the minimum is always to the right of the maximum:

$$
q_{3, \max }^{2}<q_{3, \min }^{2} ; \quad \text { so } q_{3, \min }^{2}=z_{1} .
$$

Again, we require that the minimum, $q_{3 \text {, } \min }^{2}$, be positive for diffusive instability. Additionally, a minimal condition for violating $H_{3}\left(q^{2}\right)>0$ is that

$$
H_{3}\left(q_{3, \min }^{2}\right)<0 \text {. }
$$

Notice again that in (20) at every occurrence of $q^{2}$ either $D_{1}$ or $D_{2}$ always appears in the term, and that $D_{1}$ and $D_{2}$ never appear in a term not containing $q^{2}$. This means that $H_{3}(0)$ represents condition (23c) without diffusion, and we will therefore also require $H_{3}(0)>0$.

Additionally, since

$$
q_{3, \min }^{2}=\frac{-\beta+\sqrt{\beta^{2}-3 \alpha \gamma}}{3 \alpha},
$$

and, since $q$ represents a physical wavenumber, we want $q_{3 \text {, min }}^{2}$ to be real. Therefore an additional requirement is that the radicand $\beta^{2}-3 \alpha \gamma>0$.

For $\beta^{2}-3 \alpha \gamma<0, H_{3}(z)$ has no real minimum. Since $H_{3}(0)>0$ by $(23 \mathrm{c})$ and $d H_{3} / d x$ is everywhere negative, it appears that for

$$
\frac{d H_{3}}{d x}(z=0)=\gamma<0,
$$

$H_{3}$ will eventually intersect zero at which point one has onset of Turing instability. However, this cannot in fact happen since

$$
\lim _{x \rightarrow \infty} H_{3}(z) \sim a z^{3}>0
$$

as $\alpha>0$ (note that $\gamma<0, \beta^{2}-3 \alpha \gamma<0$ implies that $d H_{3} / d z<0$ or $H_{3}(z)$ is monotonic decreasing everywhere).

The following is a summary of the conditions contributed from the cubic condition:

(a) $H_{3}(0)<0$ - unstable without diffusion; so instability is not diffusion driven;

(b) $H_{3}(0)>0, q_{3, \min }^{2}<0-$ no instability; 
(c) $H_{3}(0)>0, q_{3, \min }^{2}>0$, radicand $<0$-no instability;

(d) $H_{3}(0), q_{3, \min }^{2}>0$, radicand $>0, H_{3}\left(q_{3, \min }^{2}\right)>0$-no instability;

(e) $H_{3}(0)>0, q_{3, \text { min }}^{2}>0$, radicand $>0, H_{3}\left(q_{3, \min }^{2}\right)<0$-instability.

This leads to another set of necessary and sufficient conditions for Turing instability as follows:

$$
\begin{aligned}
\text { I. } & H_{3}(0)>0, \\
\text { II. } & q_{3, \min }^{2}>0, \\
\text { III. } & \text { radicand }>0, \\
\text { IV. } & H_{3}\left(q_{3, \min }^{2}\right)<0 .
\end{aligned}
$$

There are thus two sets of necessary and sufficient conditions that lead to diffusive instability with time delay modeled by the weak generic kernel: $(30 \mathrm{~b}-\mathrm{c})$ and $(37 \mathrm{~b}-$ d). These are completely different from the necessary and sufficient conditions for instability without delay for the general reaction-diffusion model (1) arrived at by Turing [1] and summarized in Appendix A. The structure of the Turing-unstable regime is thus strongly modified by the introduction of delays modeled by the weak generic kernel. The extent of this modification will be delineated numerically in the next section for the various models.

In Appendix B, the stability analysis of the system of equations for diffusive instability with delay modeled by the "strong" kernel shows that instability may occur if any of the conditions (B.6)-(B.8) may be satisfied for some $q^{2}>0$, while being reversed for $q^{2}=0$ (equivalent to $D_{1}=D_{2}=0$ ). Since condition (B.8) is quartic in $q^{2}$, derivation of necessary and sufficient conditions for instability from this inequality will be more involved than the analysis performed earlier in this section. We will consider an alternative bifurcation-type analysis of this condition in Sec. 4 to derive the boundary of the Turing unstable region. Conditions (B.6) and (B.7) are quadratic and cubic in $q^{2}$ and thus may be treated in analogy with the analysis earlier in this section to derive the necessary and sufficient conditions for the "strong" generic kernel. We will not pursue the details here. However, it is obvious that since necessary and sufficient conditions for Turing instability with "strong" delay may be derived from any of (B.6) to (B.8), these conditions will be completely different from conditions (A.5) and (A.1) in Appendix A without delay. Thus, the strong modification of the Turing-unstable space by the introduction of delay is not specific only to the "weak" generic model discussed earlier. However, the structure of the unstable region will depend on the particular model chosen for the delay, which in turn would be determined by the physical effect inducing the delay.

4. Turing space with and without delays. The domain in parameter space where the steady state can be diffusionally driven unstable, resulting in a spatially inhomogeneous state has been referred to as the Turing space [14]. In GM-I and GM-II the parameters are $b, c_{1}, c_{2}, \mu, \nu, D_{1}$, and $D_{2}$, which results in a seven-dimensional 
Turing space. In SJ-I and SJ-II the parameters are $a, k_{0}, k_{1}, A, B, C, D_{1}$, and $D_{2}$, resulting in an eight-dimensional Turing space. For the Schnackenberg model the parameters are $a, b$, and $D$, which is much simpler, being a three-dimensional parameter space. For the Thomas model the parameters are $a, \rho, K, \alpha, b$, and $D$, resulting in a six-dimensional space. Clearly, a map of the unstable regimes will require fixing parameter values for all but two of the parameters. In this paper, we will map out the Turing unstable regime in $\left(D_{1}, D_{2}\right)$ for parameter values for the SJ-I and SJ-II models, rather than map out an unstable region in $\left(k_{0}, C\right)$ space (for example) for various values of $D_{2} / D_{1}$, as previously done for the classical diffusive instability $[14,20]$. This enables a clearer visualization of the entire unstable regime. We will, however, subsequently discuss how one would obtain the classical space discussed in [14] and [20]. The Turing spaces for the GM-I, GM-II, Schnackenberg, and Thomas models are invariant under time delay modeled by the "weak" generic kernel; so they will not be graphed, although the invariance will be discussed.

We begin by determining the steady states for each model in parametric form and calculating the $a_{i j} s$.

Segel-Jackson model. By setting $D_{1}=n 1, D_{2}=n 2$, and using (9) and (10) in (27) we obtain the following values for SJ-I and SJ-II with the steady states $\widehat{V}=\widehat{Q}=$ $c k_{0} /\left(A B-k_{1} C\right)$ and $\widehat{P}=B k_{0} /\left(A B-k_{1} C\right)$ where $a_{31}=a, a_{32}=0$, and $a_{33}=-a$, as stated before.

\begin{tabular}{|ccc|}
\multicolumn{1}{c}{} & SJ-I & SJ-II \\
\hline$a_{11}:$ & $k_{0}+2 k_{1} \widehat{V}-A \hat{P}$ & $k_{0}+k_{1} \widehat{Q}-A \widehat{P}$ \\
\hline$a_{12}:$ & $-A \widehat{V}$ & $-A \widehat{V}$ \\
\hline$a_{13}:$ & 0 & $\widehat{V} k_{1}$ \\
\hline$a_{21}:$ & 0 & 0 \\
\hline$a_{22}:$ & $B \widehat{Q}-2 C \widehat{P}$ & $B \widehat{Q} q-2 C \widehat{P}$ \\
\hline$a_{23}:$ & $B \widehat{P}$ & $B \widehat{P}$ \\
\hline
\end{tabular}

The graphs of the Turing space for the SJ-II model were done using the following parameter values:

(a) $A=B=C=1, k_{0}=0.1, k_{1}=0.9$,

(b) $A=B=C=1, k_{0}=0.1, k_{1}=-0.1$,

(c) $A=B=C=1, k_{0}=1, k_{1}=-0.1$,

(d) $A=B=C=1, k_{0}=1, k_{1}=-1$.

Gierer-Meinhardt model. By setting $D_{1}=D_{a}, D_{2}=D_{h}$, and a (delay parameter) $=b$ (to differentiate with "activator"), and using (6) and (7) in (27) we obtain the following values for GM-I and GM-II with the steady states $\hat{a}=\widehat{Q}=c_{1} \nu /\left(c_{2} \mu\right)$ and $\hat{h}=c_{1}^{2} \nu /\left(c_{2} \mu^{2}\right)$ where $a_{31}=b, a_{32}=0$, and $a_{33}=-b$, as stated before. 
GM-I GM-II

\begin{tabular}{|ccc|}
\hline$a_{11}:$ & $\frac{c_{1} \widehat{Q}}{\hat{h}}-\mu$ & $\frac{c_{1} \widehat{Q}}{\hat{h}}-\mu$ \\
\hline$a_{12}:$ & $-\frac{c_{1} \hat{a} \widehat{Q}}{\hat{h}^{2}}$ & $-\frac{c_{1} \hat{a} \widehat{Q}}{\hat{h}^{2}}$ \\
\hline$a_{13}:$ & $\frac{c_{1} \hat{a}}{\hat{h}}$ & $\frac{c_{1} \hat{a}}{\hat{h}}$ \\
\hline$a_{21}:$ & $2 c_{2} \hat{a}$ & $c_{2} \hat{Q}$ \\
\hline$a_{22}:$ & $-\nu$ & $-\nu$ \\
\hline$a_{23}:$ & 0 & $c_{2} \hat{a}$ \\
\hline
\end{tabular}

Schnackenberg model. Using (8) in (27) we obtain the following for the steady states $\hat{u}=\widehat{Q}=a+b$ and $\hat{v}=b /(a+b)^{2}$ :

$$
\begin{array}{lll}
a_{11}=\hat{v} \widehat{Q}-1 ; & a_{12}=\hat{u} \hat{Q} ; & a_{13}=\hat{u} \hat{v} ; \\
a_{21}=-\hat{v} \widehat{Q} ; & a_{22}=-\hat{u} \widehat{Q} ; & a_{23}=-\hat{u} \hat{v} ; \\
a_{31}=a ; & a_{32}=0 ; & a_{33}=-a .
\end{array}
$$

Thomas model. Here we use (9) in (27) to obtain the specific linearized equations as follows. The steady state cannot be analytically derived and will be referred to as $\hat{u}$ and $\hat{v}$, and for this model we set $a$ (the delay parameter) $=d$ (to differentiate from parameter $a)$ :

$$
\begin{array}{llll}
a_{11}=-1+\frac{\rho Q v(1+2 K u)}{\left(1+u+K u^{2}\right)^{2}} ; & a_{12}=-\frac{\rho Q}{1+u+K u^{2}} ; & a_{13}=-\frac{\rho v}{1+u+K u^{2}} ; \\
a_{21}=\frac{\rho Q v(1+2 K u)}{\left(1+u+K u^{2}\right)^{2}} ; & a_{22}=-\alpha-\frac{\rho Q}{1+u+K u^{2}} ; & a_{23}=-\frac{\rho v}{1+u+K u^{2}} ; \\
a_{31}=d ; & a_{32}=0 ; & a_{33}=-d .
\end{array}
$$

Now we ensure that all parameter values considered result in stable systems in the absence of diffusion. In other words, conditions (30a) and (37a) (or (23)) must be satisfied for all values of $a>0$ (negative values of $a$ have no physical meaning). Conditions (23) and (30a) are easily verified. Condition (37a) results in a function of the delay parameter $a$ as follows:

$$
\begin{aligned}
H_{3}(0)= & -\left(a^{2} a_{11}\right)+a a_{11}^{2}-a^{2} a_{13}+a a_{11} a_{13}+a_{11} a_{12} a_{21} \\
& -a^{2} a_{22}+2 a a_{11} a_{22}-a_{11}^{2} a_{22}+a_{22} a_{12} a_{21}+a a_{22}^{2} \\
& -a_{11} a_{22}^{2}+a a_{12} a_{23} .
\end{aligned}
$$

$H_{3}(0)$ is shown in Fig. 1 (see p. 46) for model SJ-II with the parameter (a). It is seen to be everywhere positive, thus satisfying condition (37a). The graph of $H_{3}(0)$ versus $a$ is qualitatively very similar to this graph for all other models with various parameter sets. Thus, the inequalities (30a) and (37a) (or (23)) for stability of the steady states in the absence of delay are satisfied. 


$$
H_{3}(0) \quad \text { SJ-II (a) }
$$

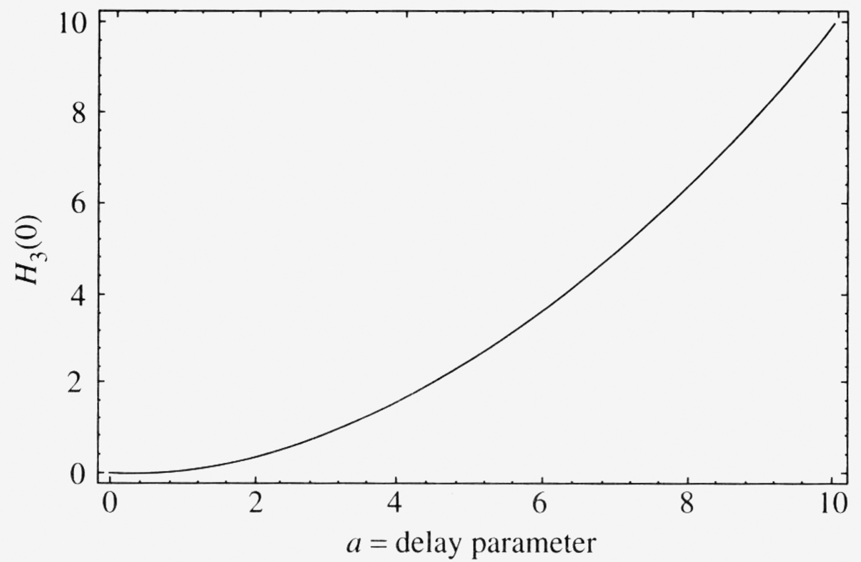

FIG. 1. $H_{3}(0)$ as a function of delay parameter $a$ for model SJ-II, with $A=B=C=1, k_{0}=0.1$, and $k_{1}=0.9$. Note that $H_{3}(0)$ is everywhere positive, so that (37a) is satisfied.

Analyzing the first set of necessary and sufficient conditions for instability, (30b) and (30c), i.e., $q_{2, \min }^{2}>0, H_{2}\left(q_{2, \min }^{2}\right)<0$, one notes that both $q_{2, \min }^{2}$ and $H_{2}\left(q_{2, \min }^{2}\right)$ are independent of $a$; so we can graph each in three dimensions as functions of the diffusion coefficients $D_{1}$ and $D_{2}$. This is shown in Figs. 2 and 3 for the SJ-II

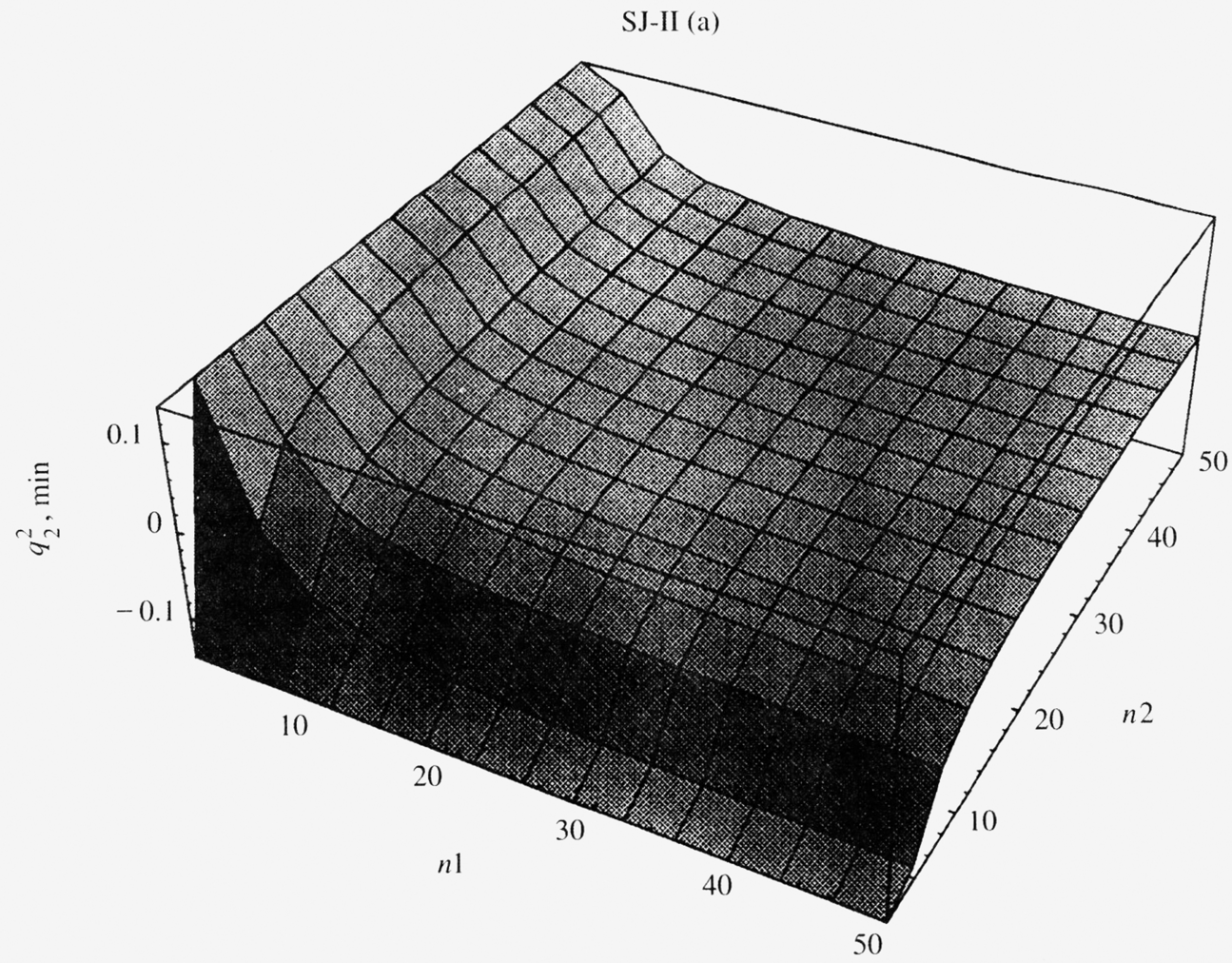

FIG. 2. The minimum $q_{2, \min }^{2}$ of (17) for model SJ-II, with $A=$ $B=C=1, k_{0}=0.1$, and $k_{1}=0.9$, as a function of the diffusion 


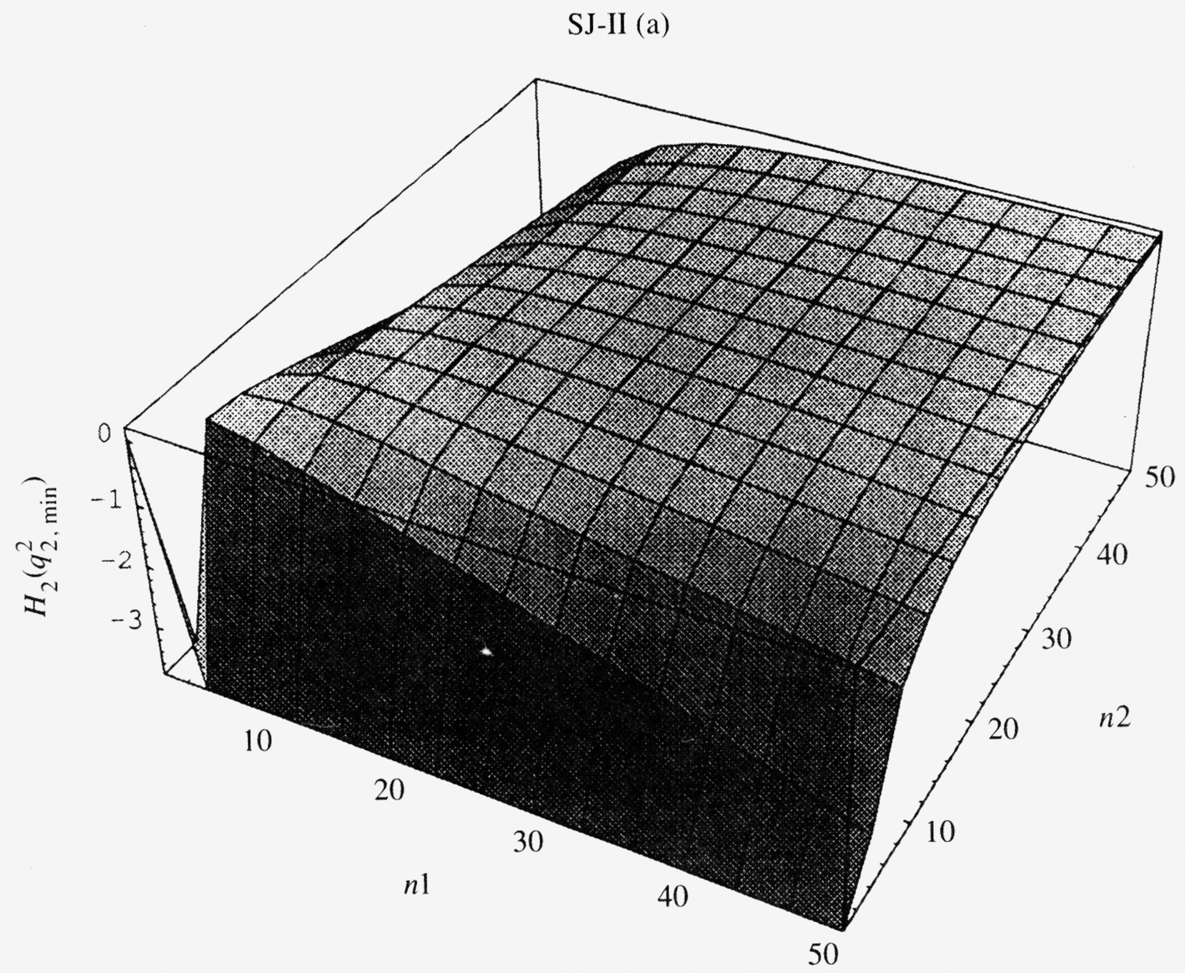

FIG. 3. The minimum value $H_{2}\left(q_{2}^{2}\right.$, min $)$ of (18) for model SJ-II, $A=$ $B=C=1, k_{0}=0.1$, and $k_{1}=0.9$, as a function of the diffusion coefficients $n 1$ and $n 2$. Note that $H_{2}\left(q_{2}^{2}\right.$, min $)<0$ everywhere.

model with parameter set (a). There is a regime of Turing instability in the region of $\left(D_{1}, D_{2}\right)$ where both inequalities $(30 \mathrm{a})$ and $(30 \mathrm{~b})$ are satisfied. Since it is difficult to visualize this Turing-unstable space by superimposing Figs. 2 and 3, we take slices of the space at $n 1\left(D_{1}\right)=1,5$, and 10 to obtain Figs. 4 to 6 (see pp. 48 and 49 ). The unstable $n 2\left(D_{2}\right)$ parameter range for each value of $n 1\left(D_{1}\right)$ can now easily be read from the graph, e.g., $n 2 \geq 3$ for $n 1=1, n 2 \geq 11$ for $n 1=5$, and $n 2 \geq 22$ for $n 1=10$, implying that the Turing space contracts as $n 1$ increases. Note that this is only a partial view of the unstable region, whereas a level curve analysis would allow visualization of the entire Turing space. By using the concept of level curves one may compress all the information on three-dimensional surfaces such as Figs. 2 and 3 onto the $\left(D_{1}, D_{2}\right)$-plane where the entire Turing-unstable space may then be clearly identified.

By performing a level curve analysis on the quadratic conditions, we are able to clearly identify (from the intersection of the regimes that satisfy the instability conditions) the parameter regime leading to diffusive instability. These level curve analyses may be applied to the classical conditions without delay making those results more transparent. First set $q_{2, \text { min }}^{2}=k_{1}$ and $H_{2}\left(q_{2, \text { min }}^{2}\right)=k_{2}$ and solve for $D_{2}$. We 


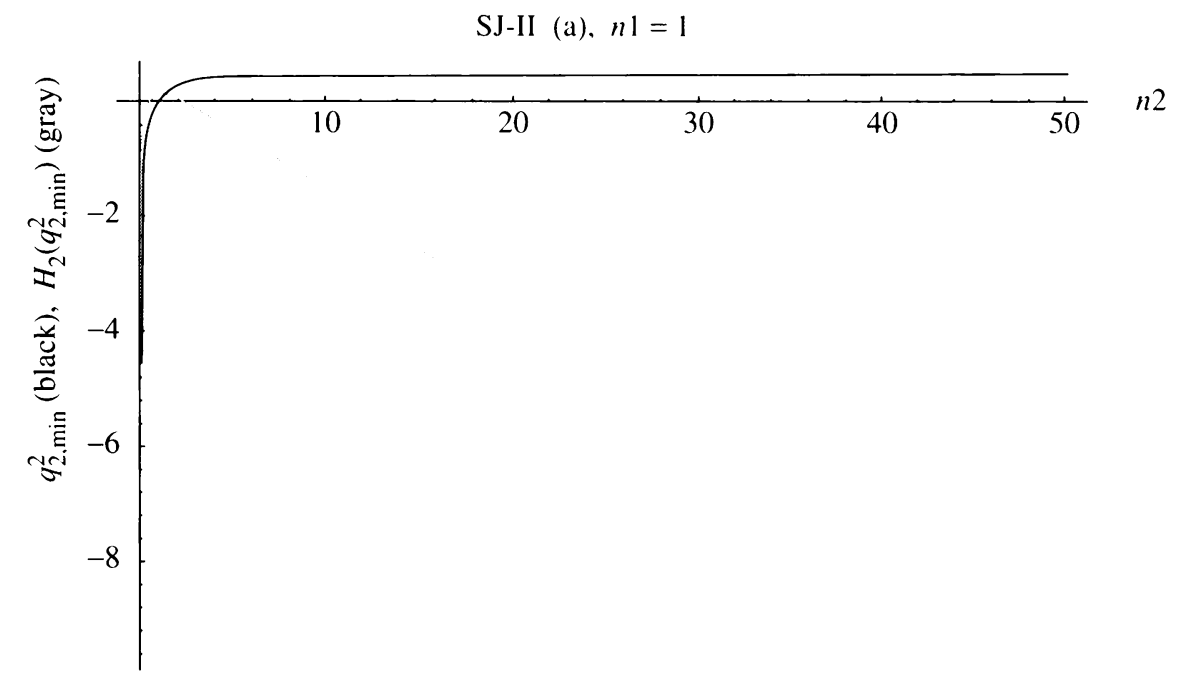

FIG. 4. The minimum $q_{2}^{2}$, min (black) and the minimum value $H_{2}\left(q_{2}^{2}\right.$, min $)$ (gray) for model SJ-II, with $A=B=C=1, k_{0}=0.1$, and $k_{1}=0.9$, as a function of $n 2$ for $n 1=1$. The diffusively unstable region satisfying (30) is $n 2 \geq 3$.

SJ-II (a), $n 1=5$

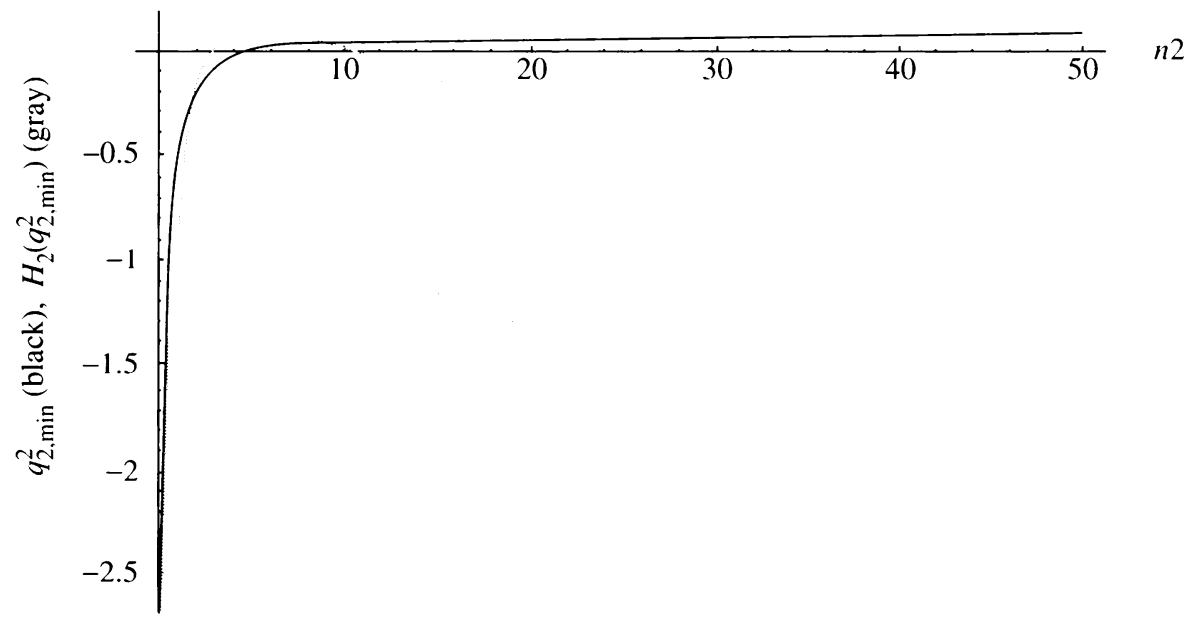

FIG. 5. The same as Fig. 4 for $n 1=5$. The Turing unstable region satisfying (30) is $n 2 \geq 11$.

obtain the following families of level curves:

$$
\begin{aligned}
& D_{2}^{(\mathrm{i})}\left(k_{1}, D_{1}\right)=\frac{D_{1} a_{22}}{2 D_{1} k_{1}-a_{11}-a_{13}}, \quad k_{1} \geq 0, \\
& D_{2}^{\text {(ii) }}\left(k_{2}, D_{1}\right)=\frac{D_{1}}{2 B}\left\{\left(k_{2}-L\right)-\sqrt{\left(L-k_{2}\right)^{2}-4 M N}\right\}, \quad k_{1} \leq 0 \text {, } \\
& D_{2}^{(\mathrm{iii})}\left(k_{2}, D_{1}\right)=\frac{D_{1}}{2 B}\left\{\left(k_{2}-L\right)+\sqrt{\left(L-k_{2}\right)^{2}-4 M N}\right\}, \quad k_{1} \leq 0 \text {, }
\end{aligned}
$$




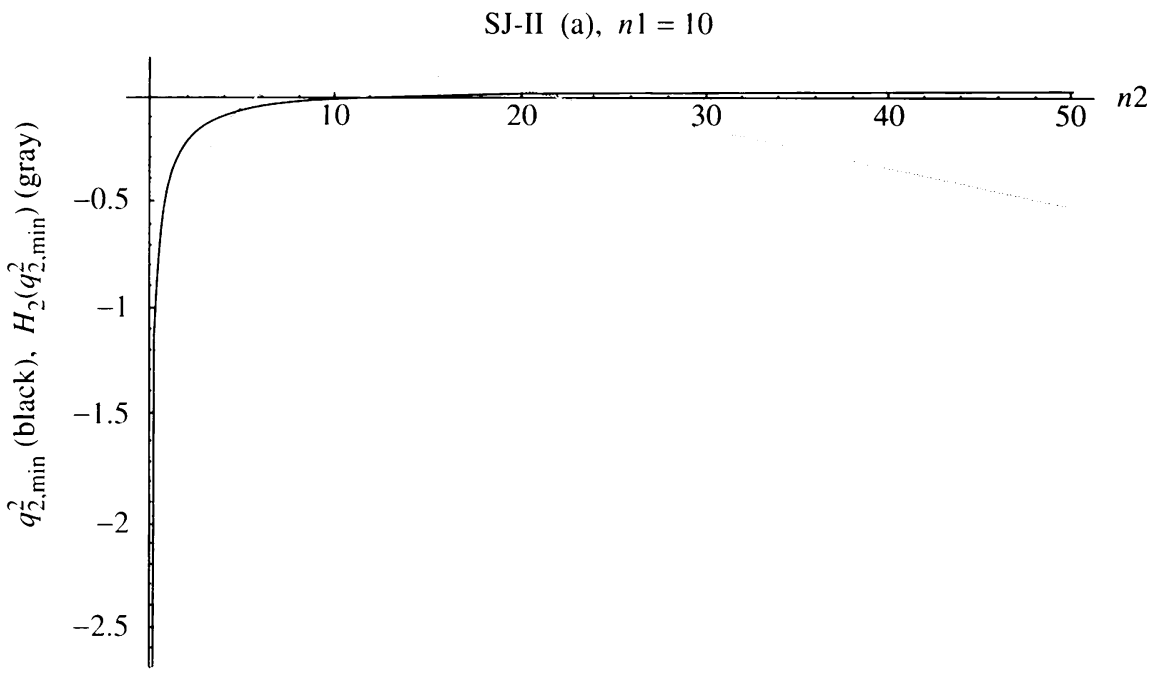

FIG. 6. The same as Fig. 4 for $n 1=10$. The Turing unstable region satisfying $(30)$ is $n 2 \geq 22$.

where

$$
\begin{aligned}
L & =-a_{12} a_{21}+\frac{a_{11} a_{22}}{2}+\frac{a_{13} a_{22}}{2}-a_{12} a_{23}, \\
M & =-\frac{a_{11}^{2}}{4}-\frac{a_{11} a_{13}}{2}-\frac{a_{13}^{2}}{4}, \\
N & =-\frac{a_{22}^{2}}{4} .
\end{aligned}
$$

Next we determine the region where $q_{2, \min }^{2}>0$ by setting $k_{1}=0,10$. Similarly, we determine the region where $H_{2}\left(q_{2}^{2}\right.$, min $) \leq 0$ by setting $k_{2}=0,-10$. By superimposing the above results in the SJ-II model with parameter set (a) we obtain the graph in Fig. 7 (see p. 50). Here the slanted black line is $D_{2}^{(\mathrm{i})}\left(0, D_{1}\right)$, the upper and lower gray curves are $D_{2}^{(\mathrm{ii})}\left(0, D_{1}\right)$ and $D_{2}^{(\mathrm{ii})}\left(-10, D_{1}\right)$, respectively, and the lower and upper dashed lines are $D_{2}^{(\text {iii) }}\left(0, D_{1}\right)$ and $D_{2}^{(i i i)}\left(-10, D_{1}\right)$, respectively. As $k_{2} \rightarrow-\infty, D_{2}^{(\text {ii) }}\left(k_{2}, D_{1}\right)$ and $D_{2}^{(\text {iii })}\left(k_{2}, D_{1}\right)$ asymptotically approach the $n 2$ and $n 1$ axes, respectively. From this we see that the regions of the $(n 1, n 2)$-plane where $H_{2}\left(q_{2, \min }^{2}\right) \leq 0$ are between the gray line and the $n 2$-axis and between the dashed line and the $n 1$-axis. Note that $D_{2}^{(i)}\left(k_{1}, D_{1}\right)$ has an asymptote at $D_{1}=\left(a_{11}+a_{13}\right) /\left(2 k_{1}\right)$ and, by using various values of $k_{1}$, we see that the region of the $(n 1, n 2)$-plane where condition $(30 \mathrm{~b})\left(q_{2, \min }^{2}>0\right)$ is satisfied (i.e., the level curves associated with $\left.k_{1} \geq 0\right)$ is the entire region above the slanted black line $D_{2}^{(i)}\left(0, D_{1}\right)$. For this level curve $D_{2}^{(\mathrm{i})}\left(k_{1}, D_{1}\right)$ there is a lower region in the fourth quadrant satisfying our conditions which we disregard since negative $D_{2}$ values do not have physical significance. 


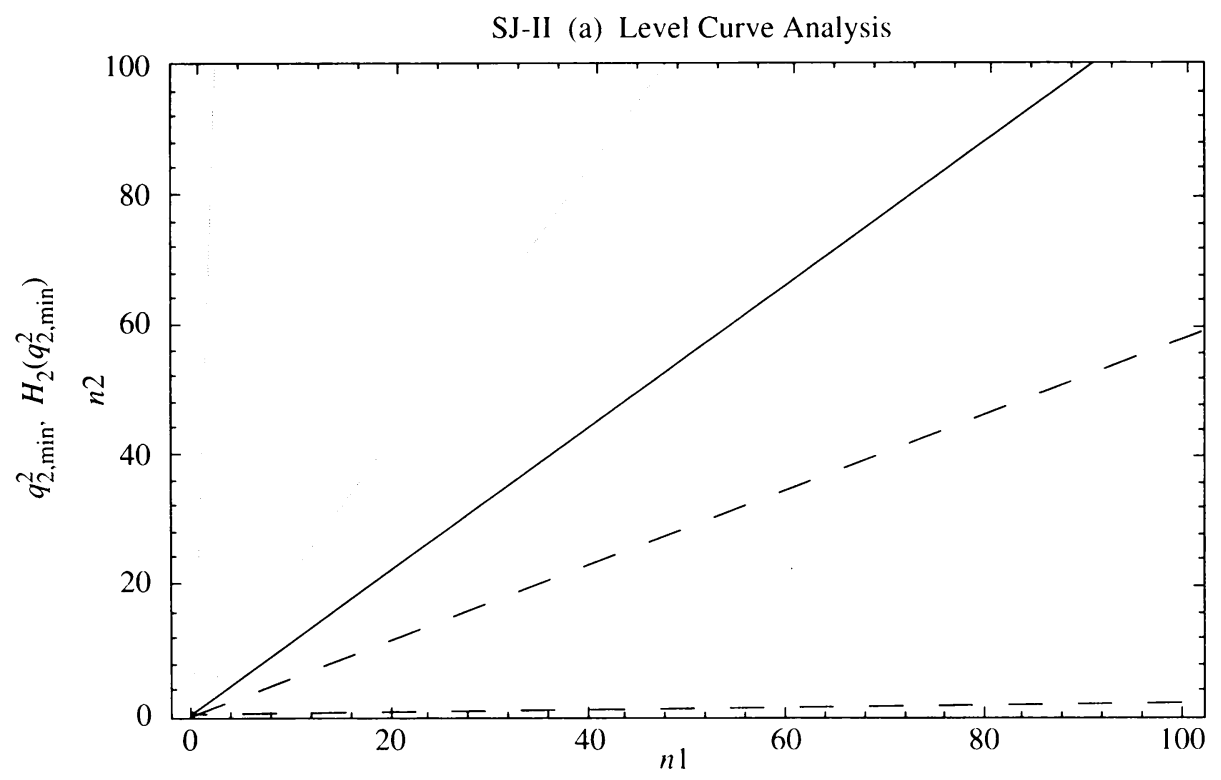

FIG. 7. The level curves $D_{2}^{(\mathrm{i})}\left(0, D_{1}\right)$ (black), $D_{2}^{(\mathrm{ii})}\left(0, D_{1}\right)$ (lower gray), $D_{2}^{\text {(ii) }}\left(-10, D_{1}\right)$ (upper gray). $D_{2}^{\text {(iii) }}\left(0, D_{1}\right)$ (upper dashed), and $D_{2}^{(\text {iii) }}\left(-10, D_{1}\right)$ (lower dashed) for model SJ-II with $A=B=$ $C=1, k_{0}=0.1$, and $k_{1}=0.9$. The Turing Space or diffusively unstable regime is the region above the lower gray line.

Clearly the Turing space is the portion of the positive $\left(D_{1}, D_{2}\right)$-quadrant above the lower gray line. We find that the regions of instability, if they exist, are qualitatively similar for all models regardless of parameter values. Thus, in the majority of cases the curve $D_{2}^{(i i)}\left(0, D_{1}\right)$ and the $D_{2}$-axis bound the Turing space.

Now, using the second set of necessary and sufficient conditions for instability (37) and recalling that (37a) is true for all of the parameter sets, we can also analyze the region of instability using level curves in $(n 1, n 2)$-space (for fixed values of the delay parameter $a$ ). We find that the cubic conditions for diffusive instability are almost never met. Very few parameter sets for any of the models, with time delays in one or both equations, resulted in $q_{3, \min }^{2}>0$. Additionally, the three-dimensional graphs of $q_{3, \min }^{2}$ as a function of $D_{1}$ and $D_{2}$ (for various delay parameters) are qualitatively similar in all cases. A typical graph is shown in Fig. 8 for GM-II with parameter values

$$
c_{1}=c_{2}=1, \quad \mu=0.1, \quad \nu=1, \quad \text { and } b=10 .
$$

An example of a model that does result in satisfying the cubic conditions for instability is the SJ-I model for the parameter set (d) above. The instability only occurs for very small values of the delay parameter $a$, i.e., when the effects of the past are strong. The three-dimensional graph of $q_{3, \min }^{2}$ in Fig. 9 (see p. 52) reveals that there is a region of $(n 1, n 2)$-space where $q_{3, \min }^{2}$ is positive, and since $H_{3}\left(q_{3, \min }^{2}\right)$ 
GM-II, $b=10$

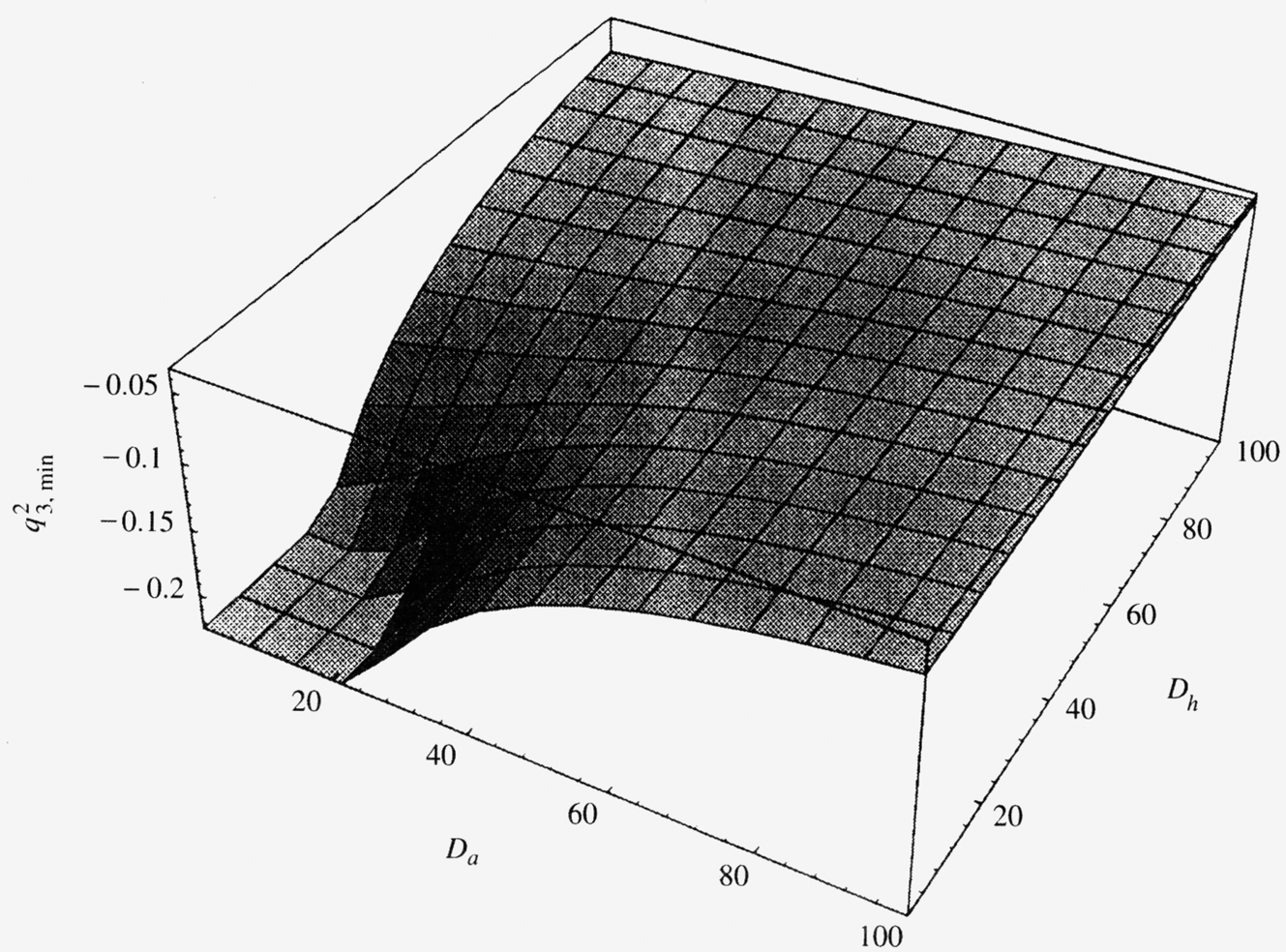

FIG. 8. The minimum $q_{3}^{2}$, min of (32) for model GM-II, with $c_{1}=$ $c_{2}=1, \mu=0.1, \nu=1$, and delay parameter $b=10$, as a function of the diffusion coefficients $D_{a}$ and $D_{h}$. Note that $q_{3, \min }^{2}<0$ everywhere, so that no diffusive instability occurs via (37). Similar behavior occurs for other $b$ values.

seems to always have a region where it is negative there is clearly a Turing space. This can be seen in Figs. 10 and 11 (see pp. 52 and 53), where the parameter is set to 0.01 . Varying $n 1$ from 0.1 to 10 results in different unstable regions. Both of these are single parameter graphs, showing $q_{3 \text {, min }}^{2}$ as a solid curve and $H_{3}\left(q_{3 \text {, min }}^{2}\right)$ as a gray curve. To maintain clarity the radicand was not graphed, since in both cases the radicand is greater than zero.

Interestingly, time delay can also modify an otherwise unstable system without diffusion to one that is stable. This occurred for the SJ-II model using the following parameter sets (b) and (c):

(b) $A=B=C=1, k_{0}=1, k_{1}=-0.1$,

(c) $A=B=C=1, k_{0}=1, k_{1}=-1$.

For both these sets of parameter values the Segel-Jackson model without delay did not satisfy the necessary conditions (10), i.e., it was unstable without diffusion, but the SJ-II model did. Upon introducing delay these parameter sets lead to systems that are stable everywhere. This becomes evident by introducing diffusion and analyzing 


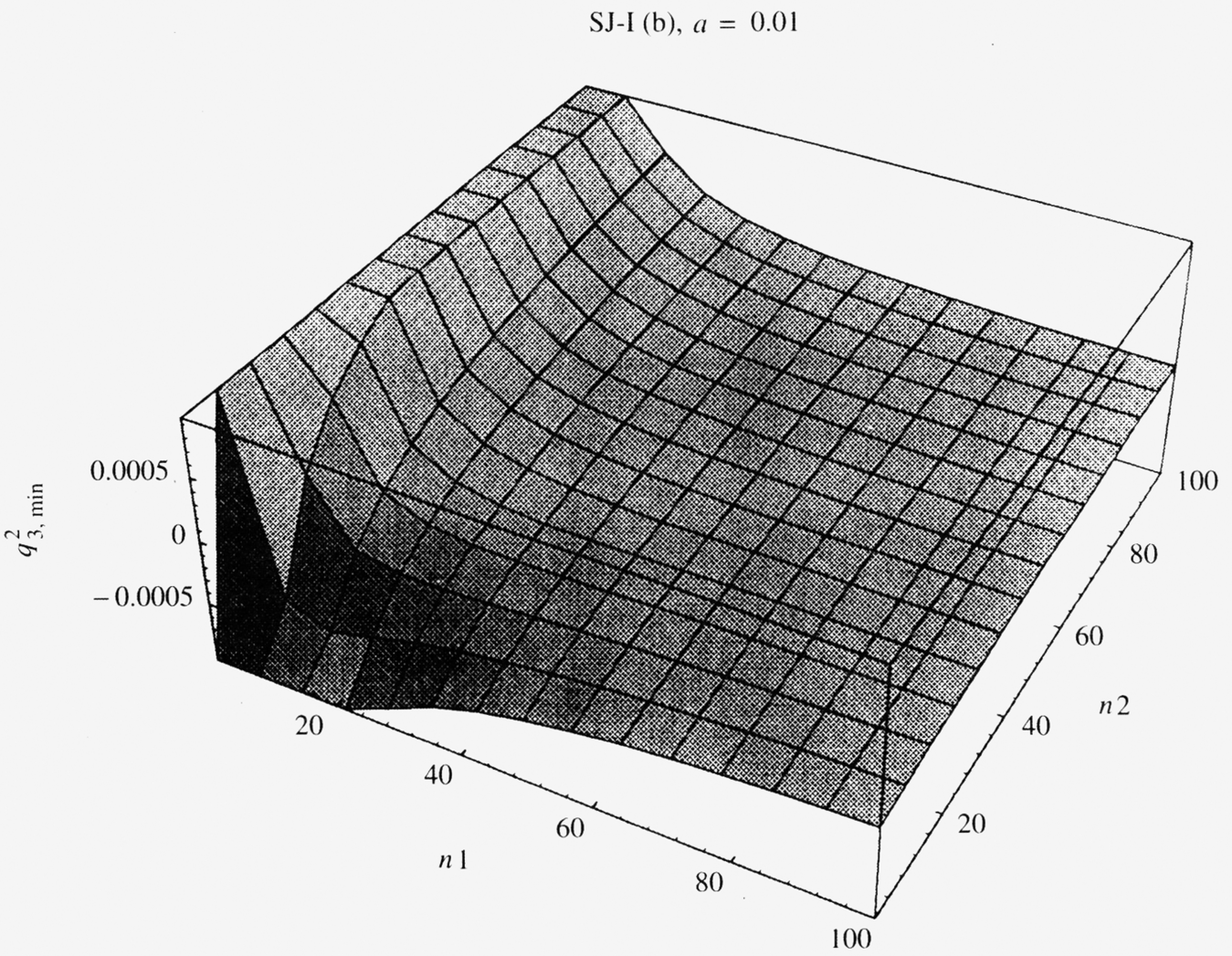

FIG. 9. The same as Fig. 8 for model SJ-I, with $A=B=C=1, k_{0}=$ $0.1, k_{1}=-0.1$, and delay parameter $a=0.01$. Now $q_{3, \min }^{2}>0$ for small values of $n 1$, so that diffusive instability may occur.

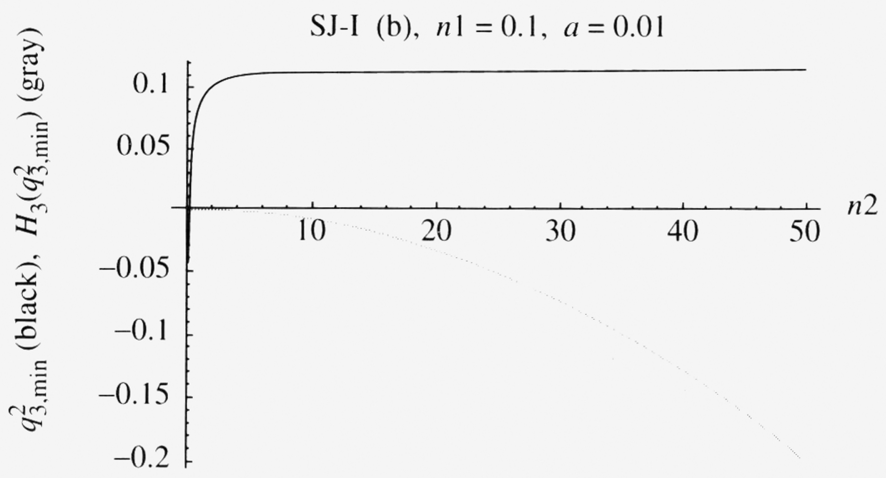

FIG. 10. The minimum $q_{3, \min }^{2}$ (black) and the minimum value $H_{3}\left(q_{3, \text { min }}^{2}\right)$ (gray) for model SJ-I, with $A=B=C=1, k_{0}=$ $0.1, k_{1}=-0.1$, delay parameter $a=0.01$, and $n 1=0.1$. There is a diffusive instability for almost all values of $n 2$. 
SJ-I (b), $n 1=10, a=0.01$

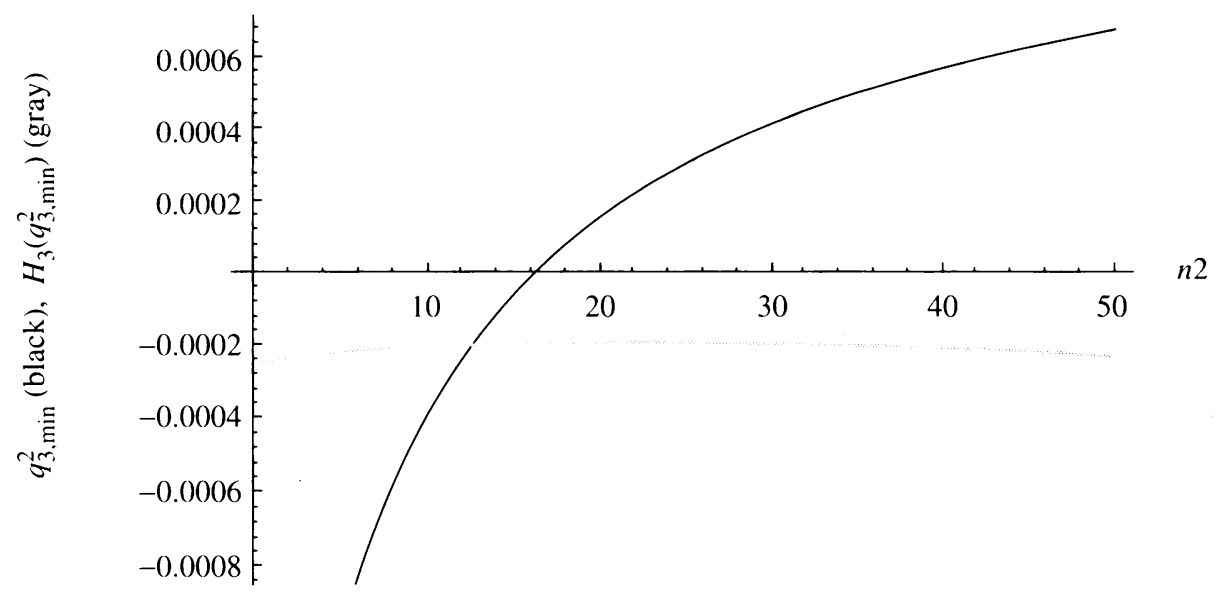

Fig. 11. The same as Fig. 10 but with $n 1=10$. The diffusively unstable region satisfying (37) is $n 2 \geq 17$.

SJ-II (c)

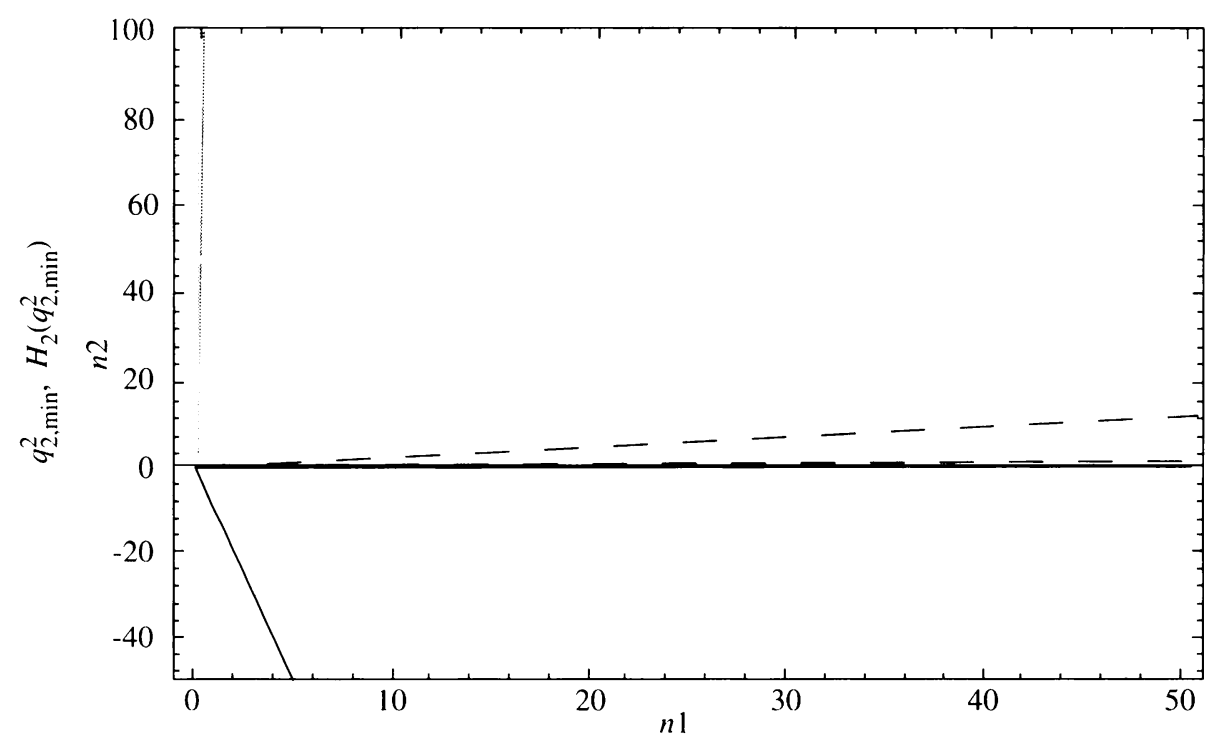

Fig. 12. The level curves $D_{2}^{(\mathrm{i})}\left(0, D_{1}\right)$ (lower black), $D_{2}^{(\mathrm{i})}\left(10, D_{1}\right)$ (upper black), $D_{2}^{(\mathrm{ii})}\left(0, D_{1}\right) \quad$ (lower gray), $D_{2}^{(\mathrm{ii})}\left(-10, D_{1}\right) \quad$ (upper gray), $D_{2}^{(\mathrm{iii})}\left(0, D_{1}\right)$ (upper dashed), and $D_{2}^{(\mathrm{iii})}\left(-10, D_{1}\right)$ (lower dashed) for model SJ-II with $A=B=C=1, k_{0}=1$, and $k_{1}=-0.1$. There is now no diffusively unstable regime satisfying (30).

the level curves associated with $q_{2, \min }^{2}=k_{1} \geq 0$. In Figs. 12 and 13 (see p. 54), corresponding to parameter sets $(\mathrm{b})$ and (c) respectively, the lower and upper black lines are $D_{2}^{(\mathrm{i})}\left(0, D_{1}\right)$ and $D_{2}^{(\mathrm{i})}\left(10, D_{1}\right)$, respectively. It turns out that the region of the $(n 1, n 2)$-plane where this condition is satisfied is the wedge in the fourth quadrant between the two black lines. Even if this were physically relevant there is 
SJ-II (d)

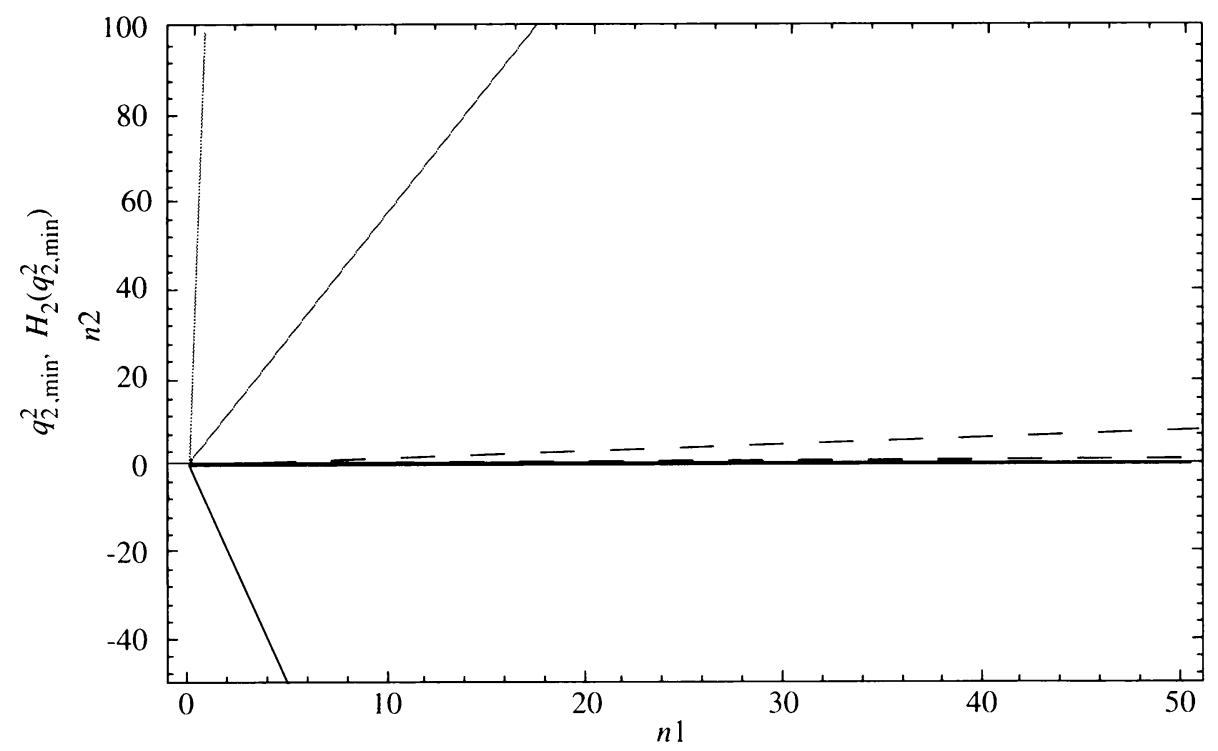

FIG. 13. The same as Fig. 12 for model SJ-II, with $A=B=C=$ $1, k_{0}=1$, and $k_{1}=-1$.

still no region of intersection where all the quadratic conditions are met. Additionally, the cubic conditions for instability are never met. Therefore, we find that in some cases time delay may be a stabilizing influence in a system, eliminating Turing-type pattern formation that existed in the absence of delay.

Comparison of the Turing space with delays modeled by the "weak" generic kernel and described above, to the classical Turing space $[1,4,20,21]$ described by conditions (A5) and (A.1) of Appendix A reveals the extent to which the Turing space is modified by the introduction of delays. The same level curve analysis can be performed on the models without delay. Consider the level curves of (A.5), i.e.,

$$
a_{11} D_{2}+a_{22} D_{1}-2 \sqrt{D_{1} D_{2}}\left(a_{11} a_{22}-a_{12} a_{21}\right)^{1 / 2}=k_{3} \geq 0 .
$$

This results in

$$
\begin{array}{ll}
D_{2}^{(\mathrm{iv})}\left(k_{3}, D_{1}\right)=D_{1}\left[\frac{\bar{M}+\sqrt{\bar{M}^{2}-a_{11}\left(a_{22} D_{1}-k_{3}\right)}}{a_{11}}\right]^{2}, & k_{3} \geq 0, \\
D_{2}^{(\mathrm{v})}\left(k_{3}, D_{1}\right)=D_{1}\left[\frac{\bar{M}-\sqrt{\bar{M}^{2}-a_{11}\left(a_{22} D_{1}-k_{3}\right)}}{a_{11}}\right], \quad k_{3} \geq 0
\end{array}
$$

where

$$
\bar{M}=\sqrt{D_{1}}\left(a_{11} a_{22}-a_{12} a_{21}\right)^{1 / 2}
$$


The $q_{\min }^{2}$ level curve turns out to have the same equations as $D_{2}^{(\mathrm{i})}\left(k_{1}, D_{1}\right)$, since $a_{13}=0$ in the absence of delay.

Unstable regions in the absence of delay may be obtained by a similar analysis as was done to obtain Fig. 7 above. Time delay can completely alter the location, size, and possibly existence of instability in a system. For the parameter set (a) above, the Segel-Jackson model without delay obtained by using (8) in (A1) turns out to never have a region of instability. This is because $q_{\min }^{2}<0$ for all values of $n 1$ and $n 2$, as seen in Fig. 14. Therefore, time delay introduced the entire region of instability described by Fig. 7 .

It is evident that time delay can significantly alter the parameter regimes leading to diffusive instability. Since the boundary of the Turing space is a straight line (see Fig. 7) in $\left(D_{1}, D_{2}\right)$-space, it may be expressed in terms of dimensionless parameters as $D_{1} / D_{2}=$ constant . Murray employed this dimensionless variable [14] to present a slightly different approach to depicting the Turing space by evaluating the equation for the boundary. As already mentioned, in most cases the boundary of the Turing

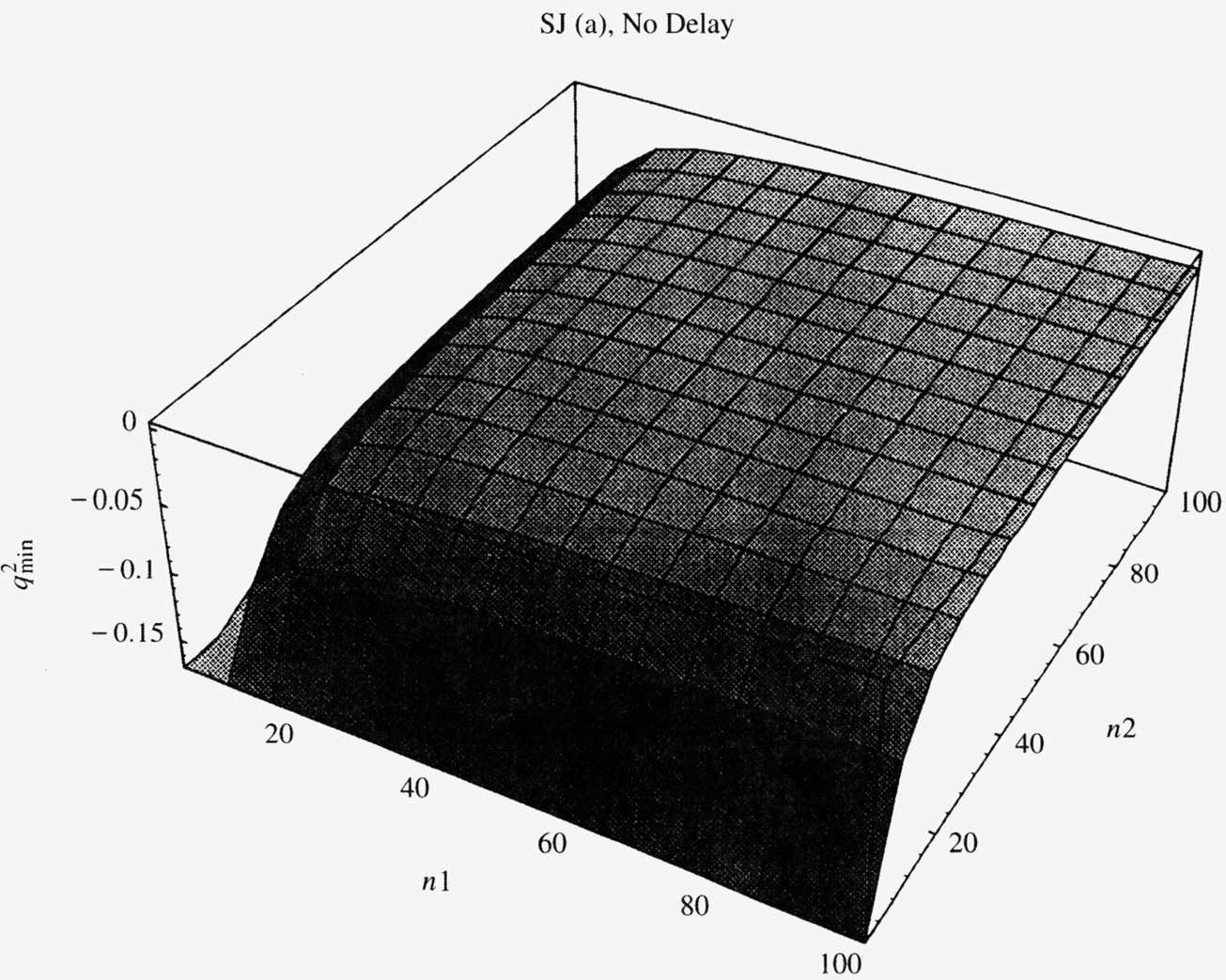

FIG. 14. The minimum $q_{\min }^{2}$ of (A6) for the Segel-Jackson model without delay and with $A=B=C=1, k_{0}=0.1$, and $k_{1}=0.9$, as a function of the diffusion coefficients $n 1$ and $n 2$. Note that $q_{\min }^{2}<0$ everywhere. 
space from the quadratic condition is given by $H_{2}\left(a_{2, \text { min }}^{2}\right)=0$. This yields

$$
D_{2}=\frac{-L+\sqrt{L^{2}-4 M N}}{2 M} D_{1} \text { or } \quad \frac{D_{2}}{D_{1}}=\frac{-L+\sqrt{L^{2}-4 M N}}{2 M},
$$

with $L, M$, and $N$ given by (39).

One may use an alternative bifurcation analysis approach to re-derive the boundaries of Turing space in (41). This provides a complement to the stability analysis in $\S 2$, and is particularly useful when dealing with more complicated cases such as the quartic condition (B.8) of Appendix B using the "strong" generic kernel. Noting that the quadratic $\mathrm{H}_{2}\left(q^{2}\right)$ of $(26)$, which introduces most of the instability, is equivalent to the coefficient $\beta_{3}$ of $(22 \mathrm{c})$, one has onset of Turing instability (via $\mathrm{H}_{2}\left(q^{2}\right)$ ) when

$$
\beta_{3}=0 \text {. }
$$

From (10), this yields $\sigma=0$ which would be useful, for instance, in weakly-nonlinear analysis of the further evolution in the vicinity of $q_{2, \min }$ which is the model that first goes linearly unstable. Solving (42) for $D \equiv D_{2} / D_{1}$ (or scaling $D_{1}$ to unity for convenience),

$$
D(q)=\frac{a_{12}\left(a_{23}+a_{21}\right)}{q^{2}\left(q^{2}-a_{11}-a_{13}\right)}+\frac{a_{22}}{q^{2}} .
$$

Treating $D$ as the bifurcation parameter [29], linear Turing instability first occurs at the extremum $q=q_{c}$ of (42). Setting $\partial D / \partial q$ to zero yields

$$
\begin{aligned}
& q_{c}^{2}=\frac{-\alpha \pm \sqrt{\alpha^{2}+a_{22}\left(a_{11}+a_{13}\right) \alpha}}{a_{22}}, \\
& \alpha=a_{12}\left(a_{21}+a_{23}\right)-a_{22}\left(a_{11}+a_{13}\right) .
\end{aligned}
$$

It is a straightforward, but tedious, calculation to show that these extrema are identical to $q_{2, \min }^{2}$ of (28) with $D=\frac{D_{2}}{D_{1}}=\frac{-L \pm \sqrt{L^{2}-4 M N}}{2 M}$ of (41). Also, the boundary of Turing space in (41) would be obtained in this alternative approach by the critical value $D\left(q_{c}\right)$ of the bifurcation parameter obtained from (43). Note that in our numerical examples, we encountered only the boundary $D=D_{c 1}=\frac{-L+\sqrt{L^{2}-4 M N}}{2 . M}$ of $(41)$, since the other boundary $D=D_{c 2}=\frac{-L-\sqrt{L^{2}-4 . M N}}{2 M}$ would yield unphysical wavenumbers $q_{c}^{2}=q_{2, \min }^{2}<0$ (as may be seen by inserting $D=D_{c 2}$ in (28) for these examples).

This approach may be employed for the cubic condition as well, although the interpretation is a little more subtle. This corresponds in the marginally unstable case to $\beta_{1} \beta_{2}-\beta_{3}=0$, with $\beta_{i}$ given by (22). Solving this for $D(q)=\frac{D_{2}}{D_{1}}(q)$, one may analogously obtain $q_{3, \min }^{2}$ from setting $\partial D / \partial q=0$, and hence obtain the boundary of Turing space from $D=D\left(q_{3, \min }\right)$. An analogous procedure would apply to each of the conditions (B.6)-(B.8) in Appendix B (for the equality or at onset of instability) using the "strong" generic kernel. This is particularly useful for the condition (B.8), which is quartic in $q^{2}$. 
The Turing space can also be depicted using (41) and fixing all parameter values except for two and varying a third value. For instance, one could plot the boundary $(41)$ in $\left(k_{0}, B\right)$-space (say) for the $\mathrm{SJ}$ models using various values for $D_{2} / D_{1}$ (which serves as parameter) with all other parameters being assigned fixed values. Since pattern formation requires long-range negative feedback (lateral inhibition), as well as positive feedback (autocatalysis), we require that the inhibitor, or prey, diffuse more rapidly $\left(D_{2}>D_{1}\right)$ [7]. In the application of the theory to the models the region of instability significantly increases when $D_{2} \gg D_{1}$. If $D=D_{2} / D_{1}$, this corresponds to a large dimensionless $D$ value, agreeing with the conclusion of Murray [14] when considering Turing space for models without time delay. Although the region of instability may be altered by the time delay, it maintains this property.

For all the models analyzed, except the SJ-II model, condition (30c) turns out to be identical to condition (A.5) in Appendix A. In other words, $H_{2}\left(q_{2, \min }^{2}\right)=H\left(q_{\min }^{2}\right)$ regardless of parameter values. As discussed above, the level curve $D_{2}^{(i i)}\left(0, D_{1}\right)$, i.e., where $H_{2}\left(q_{2}^{2}\right.$, min $)=0$, yields the boundary of the region of instability for models with delay. It turns out that the classical Turing space maintains this property, i.e., the level curve $D_{2}^{(v)}\left(0, D_{1}\right)$ defines the boundary of the unstable region for all the models described. Since most of the instability with delay is caused by the first set of necessary and sufficient conditions for instability, corresponding to the quadratic condition, the Turing space for these models is invariant with delays modeled by the weak generic kernel.

5. Summary and conclusions. The focus here was on reaction-diffusion and predator-prey models with time delay modeled by the weak generic kernel, which can lead to Turing-type diffusion-driven spatial structure. We considered several models and introduced time delays in each model. The necessary and sufficient conditions for diffusive instability with time delay were developed and applied to the models. The time delay modifies the conditions leading to diffusive instability a great deal, leading to two sets of necessary and sufficient conditions. The new conditions include a quadratic condition and a cubic condition, either of which may lead to instability. The structure of the resulting unstable parameter regimes or Turing space, where diffusion may initiate spatial pattern formation, was mapped out for four models and compared to the corresponding regimes without delay. The results indicate that in most cases the quadratic condition is the cause of the unstable region as opposed to the cubic condition. We also found that time delay modeled by the weak generic kernel may suppress instability from the model or have no effect whatsoever. The analysis performed here may be straightforwardly extended to finite domains with more than one spatial dimension $[20,21]$ by incorporating delays in the same way as done here in one dimension.

Appendix A. Turing instability in the absence of delay. In this appendix, we include the necessary and sufficient conditions for the classical Turing diffusive instability without delay for completeness. For the system (1) (with $R_{1}\left(C_{1}, C_{2}\right)$ and $R_{2}\left(C_{1}, C_{2}\right)$ not containing any delay or integral operators), the conditions analogous 
to (23) for stability of a steady state $\left(\widehat{C}_{1}, \widehat{C}_{2}\right)$ in the absence of diffusion are $[1,4$, 20, 21]

$$
\begin{array}{r}
a_{11}+a_{22}<0, \\
a_{11} a_{22}-a_{12} a_{21}>0 .
\end{array}
$$

Here,

$$
\begin{array}{ll}
a_{11}=\left.\frac{\partial R_{1}}{\partial C_{1}}\right|_{\left(\widehat{C}_{1}, \widehat{C}_{2}\right)}, & a_{12}=\left.\frac{\partial R_{1}}{\partial C_{2}}\right|_{\left(\widehat{C}_{1}, \widehat{C}_{2}\right)}, \\
a_{21}=\left.\frac{\partial R_{2}}{\partial C_{1}}\right|_{\left(\widehat{C}_{1}, \widehat{C}_{2}\right)}, \quad a_{22}=\left.\frac{\partial R_{2}}{\partial C_{2}}\right|_{\left(\widehat{C}_{1}, \widehat{C}_{2}\right)} .
\end{array}
$$

Diffusive instability may arise $[1,4,20,21]$ if

$$
a_{11}+a_{22}-D_{2} q^{2}-D_{1} q^{2}<0
$$

and/or

$$
\left(a_{11}-D_{1} q^{2}\right)\left(a_{22}-D_{2} q^{2}\right)-a_{12} a_{21}>0
$$

Analysis of these shows that no diffusive instability occurs via (A.3), while (A.4) yields the condition

$$
a_{11} D_{2}+a_{22} D_{1}>2 \sqrt{D_{1} D_{2}}\left(a_{11} a_{22}-a_{12} a_{21}\right)^{1 / 2}>0
$$

Instability first occurs via (A.4) at the wavenumber

$$
q_{\min }^{2}=\frac{a_{11} D_{2}+a_{22} D_{1}}{2 D_{1} D_{2}}
$$

Thus, (A.5) with (A.1) are the necessary and sufficient conditions for diffusive instability without delays.

Appendix B. Instability analysis with strong generic kernel. Performing the stability analysis of the system (1) using the strong generic kernel in analogy with the analysis of Sec. 2 involves [26] the introduction of another quantity $R(t)=\int_{-\infty}^{t} C_{1}(\tau) a^{2}(t-$ $\tau) e^{-a(t-\tau)} d \tau$ satisfying the linear equation

$$
\dot{R}=a(Q-R)
$$

Equation (10) is replaced by

$$
\sigma^{4}+\bar{b}_{1} \sigma^{3}+\bar{b}_{2} \sigma^{2}+\bar{b}_{3} \sigma+\bar{b}_{4}=0
$$


where

$$
\begin{aligned}
\bar{b}_{1}= & \left(D_{1}+D_{2}\right) q^{2}-a_{11}-a_{22}, \\
\bar{b}_{2}= & \left(D_{1} q^{2}-a_{11}\right)\left(D_{2} q^{2}-a_{22}\right)-a_{12} a_{21} \\
& +2 a\left(D_{1} q^{2}+D_{2} q^{2}-a_{11}-a_{22}\right)+a^{2} \\
\bar{b}_{3}= & a^{2}\left(D_{1} q^{2}+D_{2} q^{2}-a_{11}-a_{22}\right) \\
& +2 a\left[\left(D_{1} q^{2}-a_{11}\right)\left(D_{2} q^{2}-a_{22}\right)-a_{12} a_{21}\right]-a^{2} a_{13}, \\
\bar{b}_{4}= & a^{2}\left[\left(D_{1} q^{2}-a_{11}\right)\left(D_{2} q^{2}-a_{22}\right)-a_{12} a_{1}-\left(D_{1} q^{2}+D_{2} q^{2}-a_{11}-a_{22}\right)\right],
\end{aligned}
$$

and

$$
\begin{array}{ll}
a_{11}=\left.\frac{\partial R_{1}}{\partial C_{1}}\right|_{\left(\widehat{C}_{1}, \widehat{C}_{2}\right)}, & a_{12}=\left.\frac{\partial R_{1}}{\partial C_{2}}\right|_{\left(\widehat{C}_{1}, \widehat{C}_{2}\right)}, \\
a_{13}=\left.\frac{\partial R_{1}}{\partial R}\right|_{\left(\widehat{C}_{1}, \widehat{C}_{2}\right)}, & a_{21}=\left.\frac{\partial R_{2}}{\partial C_{1}}\right|_{\left(\widehat{C}_{1}, \widehat{C}_{2}\right)}, \\
a_{22}=\left.\frac{\partial R_{2}}{\partial P}\right|_{\left(\widehat{C}_{1}, \widehat{C}_{2}\right)}, & a_{23}=\left.\frac{\partial R_{2}}{\partial R}\right|_{\left(\widehat{C}_{1}, \widehat{C}_{2}\right)},
\end{array}
$$

The Routh-Hurwitz criteria [20] giving the necessary and sufficient conditions $\operatorname{Re}\left(\sigma_{i}\right)$ $<0, i=1,2,3,4$, for stability of the steady state $\left(\widehat{C}_{1}, \widehat{C}_{2}\right)$ are

$$
\bar{b}_{1}>0, \quad \bar{b}_{4}>0, \quad \bar{b}_{1} \bar{b}_{2}-\bar{b}_{3}>0, \quad \bar{b}_{1}\left(\bar{b}_{2} \bar{b}_{3}-\bar{b}_{1} \bar{b}_{4}\right)-\bar{b}_{3}^{2}>0
$$

Hence, instability may arise (for some wavenumber $q$ ) if

$$
\bar{b}_{1}<0 \text {, }
$$

and/or

$$
\bar{b}_{4}<0 \text {, }
$$

and/or

$$
\bar{b}_{1} \bar{b}_{2}-\bar{b}_{3}<0
$$

and/or

$$
\bar{b}_{1}\left(\bar{b}_{2} \bar{b}_{3}-\bar{b}_{1} \bar{b}_{4}\right)-\bar{b}_{3}^{2}<0
$$

which replace the conditions (for the "weak" generic kernel) in Sec. 2. Of these, (B.5) is linear in $q^{2}$, (B.6) a quadratic, (B.7) a cubic, and (B.8) a quartic equation in $q^{2}$. Note that one should have stability in the absence of diffusion, i.e., inequalities (B.5) $-\left(\right.$ B.8) are reversed for $D_{1}=D_{2}=0$. 
Note that the onset of instability occurs when (B.6) is just satisfied, i.e., $\bar{b}_{4}=0$ corresponds to $\sigma=0$ by (B.2). One may use the equation $\bar{b}_{4}=0$ to express $D_{2} / D_{1}$ as a function of $q$ and to obtain the critical wavenumber $q_{c}$ and $\frac{D_{2}}{D_{1}}\left(q_{c}\right)$ at the first onset of instability from $\partial\left(D_{2} / D_{1}\right) / \partial q=0$ as was done in Sec. 4 for the weak delay. The other cases of marginal instability onset, i.e., $\bar{b}_{1} \bar{b}_{2}-\bar{b}_{3}=0$ or $\bar{b}_{1}\left(\bar{b}_{2} \bar{b}_{3}-\bar{b}_{1} \bar{b}_{4}\right)=0$, may be treated similarly to derive the critical wavenumber(s) $q_{c}$ for the initial onset of the respective instabilities.

\section{REFERENCES}

[1] A. M. Turing, The chemical basis of morphogenesis, Philos. Trans. Roy. Soc. London 237, 37-72 (1952)

[2] C. W. Wardlaw, Evidence relating to the diffusion-reaction theory of morphogenesis, New Phytol. 64, 39-48 (1955)

[3] H. G. Othmer and L. E. Scriven, Instability and dynamic pattern in cellular networks, J. Theoret. Biol. 32, 507-537 (1971)

[4] L. A. Segel and J. L. Jackson, Dissipative structure: An explanation and an ecological example, J. Theoret. Biol. 37, 545-559 (1972)

[5] A. Gierer and H. Meinhardt, A theory of biological pattern formation, Kybernetik 12, 30-39 (1972)

[6] A. Gierer, Generation of biological patterns and form, Progr. Biophys. Molec. Biol. 27, 1-47 (1981)

[7] H. Meinhardt, Models of Biological Pattern Formation, Academic Press, New York, 1982

[8] M. I. Granero, A. Porati, and D. Zanacca, A bifurcation analysis of pattern formation in a diffusion governed morphogenetic field, J. Math. Biol. 4, 21-27 (1977)

[9] J. P. Keener, Activators and inhibitors in pattern formation, Stud. Appl. Math. 59, 1-23 (1978)

[10] L. A. Segel, Taxes in ecology and cell biology, Mathematical Ecology (S. A. Levin and T. Hallan, eds.), Springer-Verlag, Berlin, 1984, pp. 407-424

[11] J. Smoller, Shock Waves and Reaction-Diffusion Equations, Springer-Verlag, Berlin, 1983

[12] F. Rothe, Global Solutions of Reaction-Diffusion Systems, Springer-Verlag, Berlin, 1984

[13] P. C. Fife, Mathematical Aspects of Reacting and Diffusing Systems, Springer-Verlag, New York, 1979; E. Conway, Diffusion and predator prey interaction, in Partial Differential Equations and Dynamical Systems (W. E. Fitzgibbon, ed.), Pitman, Boston, 1984, pp. 85-133

[14] J. D. Murray, A prepattern formation mechanism for animal coat markings, J. Theoret. Biol. 88, 161-199 (1981); J. D. Murray, On pattern formation mechanisms for lepidopteran wing patterns and mammalian coat markings, Philos. Trans. Roy. Soc. London Ser. B. 295, 473-496 (1981); J. D. Murray, Parameter space for Turing instability in reaction-diffusion mechanisms: A comparison of models, J. Theoret. Biol. 98, 143-163 (1982)

[15] M. Mimura and J. D. Murray, On a diffusive predator-prey model which exhibits patchiness, J. Theoret. Biol. 75, 249-262 (1978)

[16] J. Bard, A model for generating aspects of zebra and other mamallian coat patterns, J. Theoret. Biol. 93, 363-385 (1981); A. Hunding, Bifurcations of nonlinear reaction-diffusion systems in prolate spheroids, J. Math. Biol. 17, 223-239 (1983)

[17] H. C. Schaller and H. Bodenmüller, Neurohormones and Their Functions in Hydra, Neurosecretion: Molecules, Cells, Systems (D. S. Farner and K. Lederle, eds.), Plenum, London, 1982, pp. 381-390;

W. Kemmner, A model for head regeneration in hydra, Differentiation 26, 83-90 (1984)

[18] L. Edelstein and L. A. Segel, Growth and metabolism in mycelial fungi, J. Theoret. Biol. 104, 187-210 (1983); G. Mitchison, A model for vein formation in higher plants, Proc. Roy. Soc. London Ser. B. 207, 79-109 (1980)

[19] S. A. Levin and L. A. Segel, Pattern generation in space and aspect, SIAM Review 27, 45-67 (1985)

[20] J. D. Murray, Mathematical Biology, Springer-Verlag, Berlin, 1989

[21] L. Edelstein-Keshet, Mathematical Models in Biology, Random House, New York, 1988

[22] N. MacDonald, Time lags in biological models, Lecture Notes in Biomathematics, vol. 27, SpringerVerlag, Berlin, 1978

[23] J. M. Cushing, Integrodifferential equations and delay models in population dynamic, Lecture Notes in Biomathematics, vol. 20, Springer-Verlag, Berlin, 1977 
[24] S. Roy Choudhury, On bifurcations and chaos in predator-prey models with delay, Chaos, Solitons and Fractals, vol. 2, 1992, pp. 393-409

[25] J. Hale, Theory of Functional Differential Equations, Springer-Verlag, Berlin, 1977; T. Vogel, Systemes Evolutifs, Gautier-Villars, Paris, 1965

[26] N. MacDonald, Time delay in prey-predator models, Math. Biosciences, vol. 28, 1976, pp. 321-330; N. MacDonald, Time delay in prey-predator models, II. Bifurcation theory, Math. Biosciences, vol. 33, 1977, pp. 227-234

[27] M. Farkas, Stable oscillations in a predator-prey model with time lag, J. Math. Anal. Appl. 102, 175-188 (1984)

[28] T. Myint-U and L. Debnath, Partial Differential Equations, North-Holland, New York, 1987

[29] R. K. Dodd, J. C. Eilbeck, J. D. Gibbon, and H. C. Morris, Solitons and Nonlinear Wave Equations, Academic Press, London, 1982 